\title{
Emerging treatment modalities for systemic therapy in hepatocellular carcinoma
}

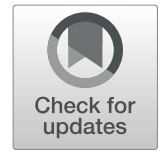

\author{
Xin Qing ${ }^{\dagger}$, Wenjing $X^{\dagger}{ }^{\dagger}$ Jingjing Zong, Xuanlong Du, Hao Peng and Yewei Zhang*
}

\begin{abstract}
Hepatocellular carcinoma (HCC) has long been a major global clinical problem as one of the most common malignant tumours with a high rate of recurrence and mortality. Although potentially curative therapies are available for the early and intermediate stages, the treatment of patients with advanced HCC remains to be resolved. Fortunately, the past few years have shown the emergence of successful systemic therapies to treat HCC. At the molecular level, HCC is a heterogeneous disease, and current research on the molecular characteristics of HCC has revealed numerous therapeutic targets. Targeted agents based on signalling molecules have been successfully supported in clinical trials, and molecular targeted therapy has already become a milestone for disease management in patients with HCC. Immunotherapy, a viable approach for the treatment of HCC, recognizes the antigens expressed by the tumour and treats the tumour using the immune system of the host, making it both selective and specific. In addition, the pipeline for HCC is evolving towards combination therapies with promising clinical outcomes. More drugs designed to focus on specific pathways and immune checkpoints are being developed in the clinic. It has been demonstrated that some drugs can improve the prognosis of patients with HCC in first- or second-line settings, and these drugs have been approved by the Food and Drug Administration or are nearing approval. This review describes targeting pathways and systemic treatment strategies in HCC and summarizes effective targeted and immune-based drugs for patients with HCC and the problems encountered.
\end{abstract}

Keywords: Hepatocellular carcinoma, Targeted therapy, Immunotherapy, Tyrosine kinase inhibitors, Immune checkpoint inhibitors, Signalling pathway

\section{Introduction}

Liver cancer is the fourth most common cause of cancer deaths worldwide [1]. Hepatocellular carcinoma (HCC) is a prominent and complex subtype of primary liver cancer, accounting for over $90 \%$ of cases [2]. Hepatectomy, liver transplantation, and ablation are potentially curative for patients diagnosed with early-stage HCC [3, 4]; however, conventional systemic chemotherapy loses its survival benefits for advanced patients [5]. It is absolutely essential to develop unique treatment modalities to better manage HCC.

\footnotetext{
* Correspondence: zhangyewei@seu.edu.cn

${ }^{+}$Xin Qing and Wenjing Xu contributed equally to this work. Department of General Surgery, Zhongda Hospital, Medical School, Southeast University, Nanjing 210009, China
}

Recently, there has been significant progress in the understanding of signalling pathways, techniques to detect tumour progression, and drugs to block pathway activity, providing opportunities to develop precise treatments [6]. Multiple aberrant pathways in HCC have been characterized such as the Ras-Raf-MAPK, PI3K-AKTmTOR, Wnt- $\beta$-catenin, and JAK-STAT pathways [710]. Drugs targeting these pathways have demonstrated encouraging survival benefits and bring considerable hope to patients with HCC [11]. Furthermore, immunotherapy has been suggested as a neopotential treatment for HCC patients [12]. Immune checkpoint inhibitors (ICIs) have been used successfully in patients with HCC [13]. These targeted and immune-based agents, including sorafenib, lenvatinib, regorafenib, and nivolumab are

(c) The Author(s). 2021 Open Access This article is licensed under a Creative Commons Attribution 4.0 International License, which permits use, sharing, adaptation, distribution and reproduction in any medium or format, as long as you give appropriate credit to the original author(s) and the source, provide a link to the Creative Commons licence, and indicate if changes were made. The images or other third party material in this article are included in the article's Creative Commons licence, unless indicated otherwise in a credit line to the material. If material is not included in the article's Creative Commons licence and your intended use is not permitted by statutory regulation or exceeds the permitted use, you will need to obtain permission directly from the copyright holder. To view a copy of this licence, visit http://creativecommons.org/licenses/by/4.0/ The Creative Commons Public Domain Dedication waiver (http://creativecommons.org/publicdomain/zero/1.0/) applies to the data made available in this article, unless otherwise stated in a credit line to the data. 
already available as clinical options for first-line or second-line treatment [14-16].

With the development of molecular medicine, systemic therapy significantly enhances quality of life and has become a major means of treatment for HCC, particularly for combination therapy and subsequent therapy $[17,18]$. However, targeted therapies are often correlated with considerable resistance and adverse events (AEs), presenting a substantial challenge to further broadening existing treatment approaches for patients with HCC [19].

This review describes important pathways in HCC and discusses recent developments in targeted therapy for these tumours, including the evolution of therapeutic modalities and available options, with the goal of specifying precise treatment protocols for HCC in future studies.

\section{Targeting pathways in HCC}

Hepatocarcinogenesis is a multielement and multistep process and is associated with aberrant activation of diverse signalling pathways (Fig. 1) involving both receptor and nonreceptor actions of tyrosine kinase proteins [20]. The exploration of tumour signal transduction has been an active area in the field of basic tumour research and is the theoretical basis for various molecular targeted drugs.

\section{Ras-Raf-MAPK pathway}

The Ras-Raf-MAPK pathway is a classic cellular signalling pathway that is generally associated with cell growth and proliferation and is located downstream of several tyrosine kinase receptors [21]. In HCC, the overexpression of several molecules in this pathway such as ras, raf-1, and ERK has been previously reported [22-24]. These variations often indicate a poor prognosis and short survival time in patients [25]. In addition, increased expression of the MAPK pathway is found in high-grade tumours [26]. Therefore, MAPK pathways may play a central role in liver carcinogenesis. This conclusion has been confirmed in preclinical trials, where specific MEK inhibitors blocked tumour angiogenesis

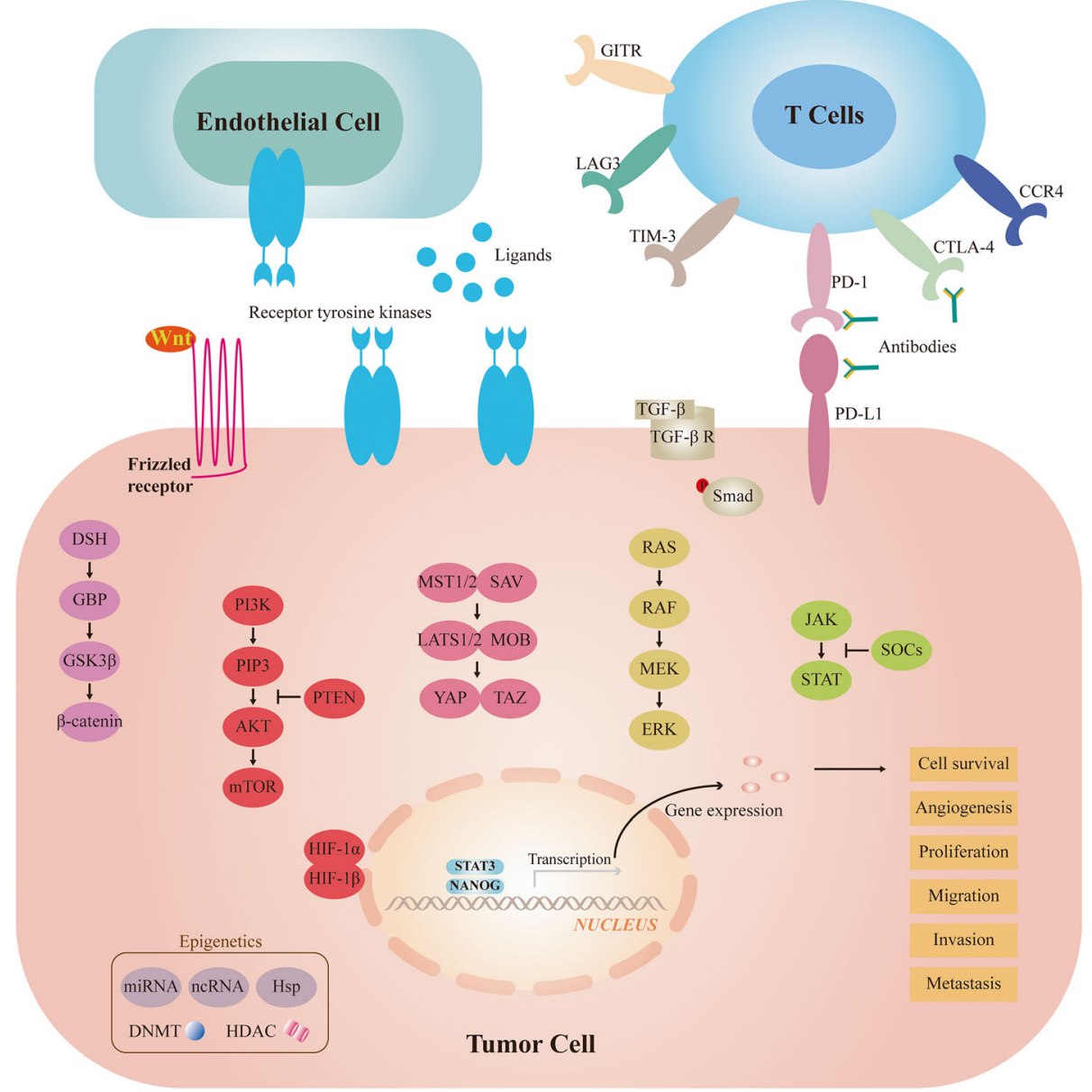

Fig. 1 Signaling pathways and therapeutic targets in focus for HCC 
and induced apoptosis by inhibiting this pathway [27]. Targeted drugs for precise molecules in this pathway have also shown promising antitumour activity in clinical trials $[28,29]$. Additionally, the HBV X protein and $\mathrm{HCV}$ core protein can also activate the Ras-Raf-MAPK pathway [30,31], and these studies indicate that this pathway may play a potential role in the progression of hepatitis to HCC.

\section{PI3K-AKT-mTOR pathways}

The PI3K-AKT-mTOR pathway plays a vital role in regulating leading cellular processes such as cell survival and metabolism, and thus its deregulation is associated with malignant transformation and recurrence, including in liver cancer [32]. The analysis of tumour tissues from HCC patients revealed that mTOR signalling activity was observed in almost 50\% of HCC samples [33]. This unrestricted activity is associated with abnormal PTEN as a tumour suppressor [34]. Notably, mutations in the PI3K-AKT-mTOR pathway are uncommon in HCC, and activation of this pathway is often largely due to liganddependent receptor activation [35]. When compared with normal samples, tumours containing aberrant levels of pathway components such as p-AKT, RICTOR, and pRPS6 are usually of a higher grade and have a worse overall prognosis [36, 37]. Moreover, cholangiocarcinoma, the second most prevalent primary liver cancer, also shows an upregulation of this pathway [38]. Some studies have demonstrated that a further increase in mTOR activation was identified in advanced HCC and in larger tumours [39]. Aberrant mTOR activation involves the PI3K-AKT and MAPK-ERK signalling pathways, which are often coinduced in HCC [40]. It has been reported that the blockade of both pathways boosts the response of HCC cells to sorafenib [41]. In addition, the PI3K-AKT-mTOR pathway is the cornerstone of the runaway signalling network in $\mathrm{HCC}$ and is a predominant driver in sorafenib resistance [42]. mTOR inhibitors are beneficial for active HCC patients after liver transplantation [43]. Various drugs targeting this pathway have shown excellent antitumour effects, particularly in combination with MAPK pathway inhibitors.

\section{Wnt- $\beta$-catenin pathways}

Activation of the Wnt- $\beta$-catenin pathway in HCC is strongly related to early development, poorly differentiated tumours, earlier recurrence, and an adverse prognosis of HCC $[44,45]$. Wnt functions as a regulator in liver regeneration and the self-renewal of progenitor and pluripotent stem cells and may be an optimal target for precise therapy [46]. Forty percent of HCC patients exhibit altered Wnt- $\beta$-catenin signalling, and nearly $20 \%$ of these mutations are caused by the $\beta$-catenin gene itself $[47,48]$. The tissue analysis of HCC samples has demonstrated that the activation of $\beta$-catenin participates in abnormal cellular proliferation, tumour metastasis, and vascular invasion [49]. Hepatitis $B$ and $C$ virus infections induce overexpression of $\beta$-catenin, thus promoting hepatocarcinogenesis $[50,51]$.

\section{JAK-STAT pathways}

In both normal and tumour cells, the JAK-STAT pathway serves multiple essential biological functions. Abnormalities of JAK-STAT can be found in over $45 \%$ of liver cancers [52]. In HCC, aberrantly activated JAKSTAT signalling results in the malfunction of downstream target genes, thereby controlling survival, cell division, angiogenesis, and metastasis [53]. The activation of STATs in tumours is considerably higher than that in adjacent liver tissues, and this degree of activation is responsible for the poor prognosis of tumour patients [54]. The JAK-STAT pathway is also responsible for the preservation of cancer stem cells with tumour propagation capacity in HCC and the establishment of an immunosuppressive microenvironment [55]. Given the oncogenic activity of JAK-STAT activation, particularly in the absence of a STAT3 disorder, targeting this pathway appears to be a favourable strategy for treating HCC.

\section{Hippo-YAP pathways}

The Hippo-YAP pathway has critical roles in controlling organ size and tissue homeostasis. However, deregulation of Hippo-YAP signalling has been demonstrated in multiple cancers and leads to various oncogenic effects [56]. Signalling is activated not only through the interaction between appropriate extracellular ligands and their cellular receptors but also via cell polarity and adhesion [57]. The expression levels of the YAP protein and mRNA were very different in normal and cancerous liver tissues, and excessive activation of YAP in mice led to hepatocellular carcinogenesis [58]. Elevated YAP levels are an early event in the development of HCC and are largely attributed to gene amplification and posttranscriptional regulation [59]. Notably, nearly $50 \%$ of HCC patients display altered YAP overexpression and nuclear localization [60], and detection of the components of Hippo-YAP signalling may provide prognostic value for patients with HCC, as the amount of nuclear YAP is highly correlated with the survival time of patients [61].

\section{Proangiogenic pathways}

$\mathrm{HCC}$ is a highly vascularized tumour, and angiogenesis is responsible for its tumorigenesis. Proangiogenic factors such as VEGF, FGF, PDGF, and hepatocyte growth factor trigger endothelial cell tyrosine kinases and downstream intracellular signalling via Ras-Raf-mTOR-Wnt pathways to activate angiogenesis [62]. These factors are 
expressed on endothelial cells and stimulate tumour neoangiogenesis, invasion, and migration. VEGF receptor expression is upregulated in HCC specimens, and elevated levels are negatively correlated with the overall survival (OS) of patients [63]. FGF is overexpressed in HCC compared with normal tissues, and this variation is recognized as oncogenic activity [64]. Dysregulation of PDGF resulting in tumorigenesis has been identified in several tumours, including HCC, and PDGF expression is markedly increased in highly metastatic cancer [65]. EGFR and IGFR are similar tyrosinase receptors, except that they have less impact on angiogenesis.

\section{Treatment advances in systemic therapy}

The occurrence of HCC is related to the complex interaction between signal transduction pathways, the tumour microenvironment, and the genetic background, which leads to many opportunities for precise treatment. The treatment regimens for HCC have undergone a major transformation with the investigation of the molecular mechanism, and many new treatment approaches have become available as therapeutic options (Fig. 2). Tyrosine kinase inhibitors (TKIs) and ICIs have improved the quality of life and greatly extended the survival time of patients with HCC. Table 1 provides a list of clinical trials evaluating systemic therapeutic agents in HCC.

\section{Targeted therapy}

Within the multitude of molecules known to be growth promoters, members of the family of protein tyrosine kinases have a central role in regulating tumour-initiating activity [66], and this is the main object of targeted therapy.
Since the availability of sorafenib as an antiangiogenic and antiproliferative agent in 2007, TKIs have revolutionized the management of $\mathrm{HCC}$, ushering in the epoch of systemic therapies. Sorafenib prolonged the median overall survival (mOS) of patients with advanced HCC from 8 to 11 months with a tolerated toxicity profile [67]. In a subsequent trial, this endpoint was further extended to 14.7 months [68]. However, no additional valid systemic treatment alternatives have been identified for nearly a decade after the introduction of sorafenib. In recent years, several novel treatment compounds have demonstrated benefits as first- and second-line regimens. New eligible agents, such as lenvatinib in the first-line setting and regorafenib, cabozantinib, and ramucirumab in the second-line setting, have already been demonstrated to improve clinical outcomes, although the mOS remains at approximately one year [69, 70]. With all of these drugs, the study design was similar to that of sorafenib, which typically selected patients with advanced $\mathrm{HCC}$ in stage $\mathrm{B}$ or $\mathrm{C}$ BCLC and assessed OS as the primary endpoint (Table 1) [14, 71]. To date, TKIs are effective treatments that have received widespread support for the treatment of advanced HCC worldwide.

Interestingly, the drugs showing significant clinical effects are almost all multikinase inhibitors (MKIs), which may be attributed to the multiple activation mechanism of hepatocellular carcinogenesis [72]. If patients with advanced HCC have failed locoregional treatment or cannot receive a liver transplant, MKIs are considered acceptable treatment agents. Certainly, if the goal is a driver mutation, the role of a single-target inhibitor may be primarily beneficial [73], but several single-target TKIs have been tested in HCC without positive results,

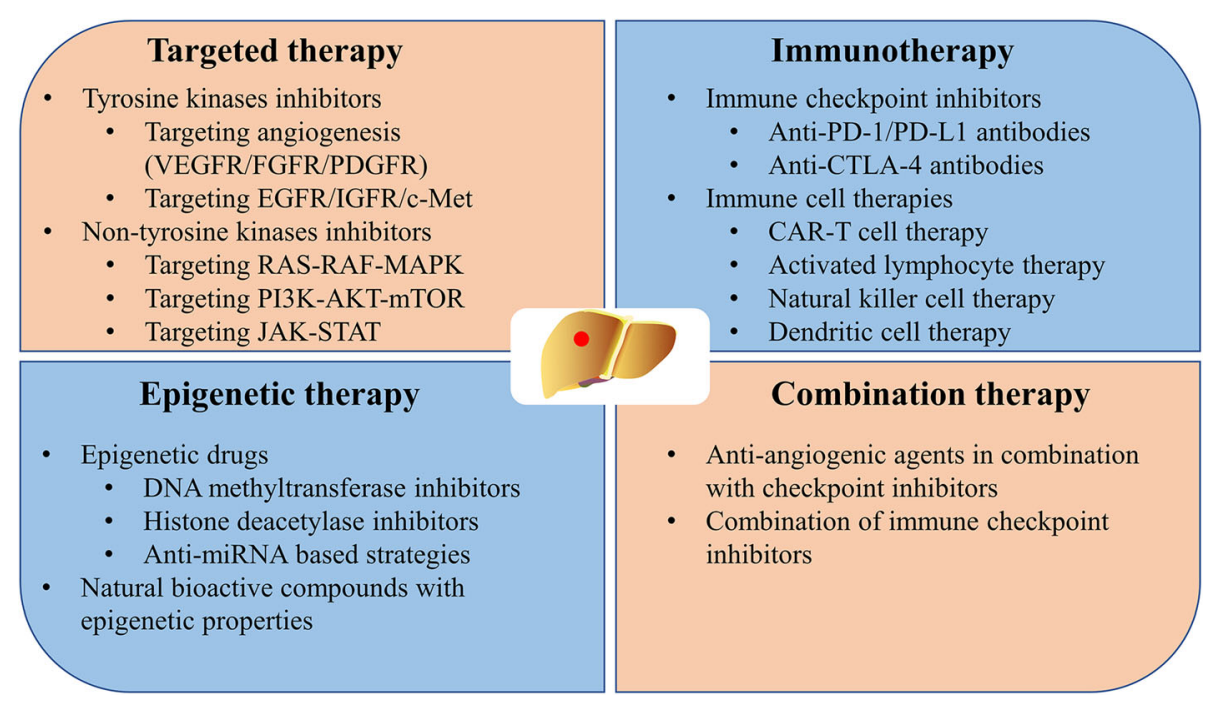

Fig. 2 Systemic treatment strategies in HCC 
Table 1 Clinical trials of systemic therapy in HCC

\begin{tabular}{|c|c|c|c|c|c|c|}
\hline Therapy & Time & Trial & Line & Drug & Control & Primary outcomes (HR; 95\% Cl) \\
\hline \multicolumn{7}{|c|}{ Targeted therapy } \\
\hline & 2005 & NCT00033462 & First & Erlotinib & None & 6-month PFS rate: $32 \%$ \\
\hline & 2007 & SHARP & First & Sorafenib & Placebo & OS: 10.7 vs. 7.9 (0.69; 0.55-0.87) \\
\hline & 2008 & Asia-Pacific & First & Sorafenib & Placebo & OS: 6.5 vs. $4.2(0.68 ; 0.50-0.93)$ \\
\hline & 2011 & NCT00699374 & First & Sunitinib & Sorafenib & OS: 7.9 vs. 10.2 (1.3; 1.13-1.5) \\
\hline & 2011 & NCT00604721 & First & Selumetinib & None & TTP: 2 \\
\hline & 2012 & BRISK-FL & First & Brivanib & Sorafenib & OS: 9.5 vs. 9.9 (1.06; 0.93-1.22) \\
\hline & 2012 & BRISK-PS & Second & Brivanib & Placebo & OS: 9.4 vs. 8.2 (0.89; 0.69-1.15) \\
\hline & 2013 & NCT00427973 & First & Cediranib & None & 3-month PFS rate: $77 \%$ \\
\hline & 2014 & EVOLVE-1 & Second & Everolimus & Placebo & OS: 7.6 vs. $7.3(1.05 ; 0.86-1.27)$ \\
\hline & 2015 & NCT01210495 & Second & Axitinib & None & 16-week DCR rate: $42.3 \%$ \\
\hline & 2015 & $\mathrm{REACH}$ & Second & Ramucirumab & Placebo & OS: 9.2 vs. 7.6 (0.87; 0.72-1.05) \\
\hline & 2016 & NCT01232296 & First & Dovitinib & Sorafenib & OS: 8.0 vs. 8.4 (1.27; 0.90-1.79) \\
\hline & 2017 & RESORCE & Second & Regorafenib & Placebo & OS: 10.6 vs. $7.8(0.63 ; 0.50-0.79)$ \\
\hline & 2018 & REFLECT & First & Lenvatinib & Sorafenib & OS: 13.6 vs. 12.3 (0.95; 0.79-1.06) \\
\hline & 2018 & NCT01004003 & First & Nintedanib & Sorafenib & TTP: 5.5 vs. $4.6(1.44 ; 0.81-2.57)$ \\
\hline & 2018 & NCT01915589 & First & Refametinib & None & ORR: 0\%; DCR: 56.3\% \\
\hline & 2018 & METIV-HCC & Second & Tivantinib & Placebo & OS: 8.4 vs. 9.1 (0.97; 0.75-1.25) \\
\hline & 2019 & CELESTIAL & Second & Cabozantinib & Placebo & OS: 10.2 vs. $8.0(0.76 ; 0.63-0.92)$ \\
\hline & 2019 & $\mathrm{REACH}-2$ & Second & Ramucirumab & Placebo & OS: 8.5 vs. $7.3(0.71 ; 0.531-0.949)$ \\
\hline & 2020 & NCT02645981 & First & Donafenib & Sorafenib & OS: 12.1 vs. 10.3 (0.831; 0.699-0.988) \\
\hline & 2020 & NCT02329860 & Second & Apatinib & Placebo & OS: 8.7 vs. $6.8(0.785 ; \mathrm{NA})$ \\
\hline \multicolumn{7}{|c|}{ Immunotherapy } \\
\hline & 2013 & NCT01008358 & First & Tremelimumab & None & PR: 17.6\%; DCR: 76.4\% \\
\hline & 2017 & Checkmate-040 & Second & Nivolumab & None & ORR: 17\% \\
\hline & 2018 & KEYNOTE-224 & Second & Pembrolizumab & None & ORR: $17 \%$ \\
\hline & 2019 & Checkmate-459 & First & Nivolumab & Sorafenib & OS: 16.4 vs. 14.7 (0.85; 0.72-1.02) \\
\hline & 2019 & KEYNOTE-240 & Second & Pembrolizumab & Placebo & PFS: 3.0 vs. 2.8 (0.718; 0.570-0.904) \\
\hline & 2020 & NCT02989922 & Second & Camrelizumab & None & ORR: $14.7 \%$ \\
\hline
\end{tabular}

OS, overall survival; PFS, progression-free survival; DCR: disease control rate; TTP: time to progression; PR: partial response; ORR: object response rate; HR, hazard ratio; $\mathrm{Cl}$, confidence interval

* Primary outcomes (months or rate)

including erlotinib, refametinib, and selumetinib [7476].

Nevertheless, targeted therapies are correlated with AEs, modest efficacy, and regular occurrence of drug resistance. Fortunately, breakthroughs in immunotherapy have brought fresh hope to HCC patients.

\section{Immunotherapy}

The object of immunotherapy is the immune system and tumour microenvironment of patients, rather than directly killing or interfering with tumour cells [77]. Common immune checkpoints in the tumour are PD-1/PDL1 and CTLA-4. The association between PD-1 and HCC was first seen in patients with HBV infection, where higher PD-1 expression was associated with an increased viral load and a fold-increased risk of HCC [78]. There are various monoclonal antibodies approved by the FDA for use in the clinic: nivolumab, pembrolizumab, and camrelizumab for anti-PD-1 and atezolizumab, aveluma, and durvalumab for anti-PD-L1 [79]. Nivolumab is the first ICI approved as a second-line therapy by the FDA to treat advanced HCC based on results from the CheckMate-040 trial [80]. Subsequently, pembrolizumab and camrelizumab also received regulatory approval in advanced HCC patients treated with sorafenib [81, 82]. Accordingly, nivolumab and pembrolizumab may be particularly beneficial for patients who are not suitable for TKIs [83]. Although CTLA-4 inhibitors have been available for solid tumours, they have not been adequately demonstrated to improve survival for treating 
this malignancy [84]. Common anti-CTLA-4 antibodies such as ipilimumab and tremelimumab are currently used in combination with anti-PD/anti-PD-L1 in the treatment of liver cancer. Nevertheless, immunotherapy is only approved for a limited number of cancer patients for various reasons, and it is necessary to explore additional immune checkpoints beyond PD-1/PD-L1.

In some tumour patients, ICIs do not elicit tumourspecific T-cell activity and are less effective in treating patients. However, this effect can be improved by cell therapy [85]. Cell therapy, such as CAR-T, TCR-T or CAR-NK, is an emerging field of treating malignant tumours. Several cell therapies for HCC have already successfully entered clinical trials and have presented excellent experimental results [86-89]. Therefore, cell therapies can significantly prevent the recurrence of $\mathrm{HCC}$ and represent a powerful treatment approach for HCC.

With the improvement of immunotherapy and the positive effects of immunotherapeutic drugs in the treatment of HCC, interest in adjuvant immunotherapy after HCC resection has increased. Immunologic drugs such as ${ }^{131}$ I-metuximab significantly improved the survival outcomes of patients after hepatectomy [90]. The rate of pathologic complete response was $29 \%$ in a phase II study evaluating nivolumab with or without ipilimumab in patients with resectable HCC [91]. This encouraging result has yet to be confirmed in the ongoing phase III trial. Neoadjuvant immunotherapy with nivolumab plus ipilimumab has demonstrated antitumour activity in an early phase trial [92]. Cytokine-induced killer (CIK) cell immunotherapy for early HCC brings a median RFS benefit of 14 months but lacks survival advantages in advanced HCC patients as an adjuvant therapy, but this result can also be enhanced by targeting myeloid-derived suppressor cells [93, 94]. A similar encouraging adjuvant immunotherapy is HSP70 mRNA-transfected dendritic cell therapy [86].

Clinical trials involving more ICIs for HCC are under investigation, including trials targeting transforming growth factor- $\beta$ (TGF- $\beta$ ), $T$ cell immunoglobulin and mucin-containing protein 3 (TIM-3), V-domain Ig suppressor of $\mathrm{T}$ cell activation (VISTA), $\mathrm{T}$ cell $\mathrm{Ig}$ and immunoreceptor tyrosine-based inhibitory motif domain (TIGIT) and lymphocyte activation gene-3 (LAG-3).

\section{Epigenetic therapy}

Sequential epigenetic alterations in regulatory genes can lead to oncogene activation and inactivation or the loss of oncogenes, resulting in tumorigenesis [95]. Major elements of epigenetic mechanisms such as ncRNAs, DNA methylation, and histone modifications change their expression or function in the background of liver fibrosis, cirrhosis, and HCC [96]. Epigenetic dysregulation represents an important function in the aetiology of HCC, which has recently been widely recognized [97]. Generally, epigenetic alterations are largely intrinsically reversible and can be seen as therapeutic targets to block the progression of HCC. Numerous drugs that specifically target epigenetic modifiers have been developed and proven successful against multiple tumour models, and several of these are in clinical trials $[98,99]$.

\section{Combination therapy \\ Anti-angiogenic agents in combination with checkpoint inhibitors}

For patients with solid tumours, vascular abnormalities can enable the tumour to evade surveillance and attack by the immune system, and these abnormalities arise from elevated angiogenic factors such as VGEF and angiopoietin 2 (ANG2). Agents targeting these molecules can normalize tumour vasculature, which in turn enhances tissue perfusion and tumour infiltration by immune cells, thereby improving the response to immunotherapy [100]. Additionally, preclinical evidence demonstrates that immunotherapy can improve the immunomodulatory effects of antiangiogenic therapy, as has been well illustrated in the available trial data [101]. Multiple clinical trials taking this into account have achieved the desired success. The combination of atezolizumab and bevacizumab (antiVEGF) is intended to change the treatment landscape, as it is the first treatment to show superiority in front-line therapy for advanced HCC in recent years. It now appears to have an overwhelming advantage over the FDAapproved first-line options of sorafenib and lenvatinib [102]. Although specific VEGF/VEGFR inhibitors may enable antitumour immunity, multikinase inhibitors remain nonnegligible agents. Lenvatinib combined with pembrolizumab is another combination therapy for advanced HCC, and early data shows good prospects [103]. Lenvatinib plus AK104 (PD-1/CTLA-4 bispecific antibody) as a first-line therapy for unresectable HCC has shown promising antitumour activity and an acceptable safety profile [104]. This study was presented at the ASCO 2021 meeting and presents a few new ideas. Camerzumab (SHR1210) in combination with apatinib has shown promising antitumour activity for HCC in a phase II study [105]. ICIs will probably become the backbone of systemic combination therapy for patients with HCC, but needs further study. The synergistic effects of ICIs and antiangiogenic drugs are being investigated in clinical trials (Table 2). This combination therapy represents an evolution of the present treatment options, which are supported by a powerful preclinical rationale.

\section{Combination of immune checkpoint inhibitors}

Because tumour escape mechanisms involve the aberrant expression of different immune checkpoint molecules, 
Table 2 Ongoing trials investigating the combination therapy of TKIs and ICls

\begin{tabular}{|c|c|c|c|c|c|c|}
\hline Targeted therapy & Checkpoint inhibitor & Phase & Patients No. & Clinical setting & Status & NCT numbers \\
\hline Lenvatinib & Nivolumab & I & 30 & HCC without prior systemic therapy & Active, not recruiting & NCT03418922 \\
\hline Lenvatinib & Nivolumab & $\|$ & 50 & Multinodular, advanced HCC & Recruiting & NCT03841201 \\
\hline Lenvatinib & Pembrolizumab & I & 104 & $\mathrm{HCC}$ & Active, not recruiting & NCT03006926 \\
\hline Lenvatinib & Pembrolizumab & III & 750 & Advanced HCC & Active, not recruiting & NCT03713593 \\
\hline Regorafenib & Nivolumab & $|/| \mid$ & 60 & HCC progressing under sorafenib & Recruiting & NCT04170556 \\
\hline Regorafenib & Nivolumab & $\|$ & 42 & Unresectable HCC & Recruiting & NCT04310709 \\
\hline Regorafenib & Nivolumab & III & 496 & Intermediate-Stage HCC & Not yet recruiting & NCT04777851 \\
\hline Regorafenib & Pembrolizumab & I & 57 & $\begin{array}{l}\text { Advanced liver cancer without prior } \\
\text { systemic therapy }\end{array}$ & Active, not recruiting & NCT03347292 \\
\hline Regorafenib & Pembrolizumab & $\|$ & 119 & Advanced or metastatic HCC after ICls & Recruiting & NCT04696055 \\
\hline Cabozantinib & Nivolumab & I & 15 & $\begin{array}{l}\text { Locally advanced HCC after definitive } \\
\text { resection }\end{array}$ & Active, not recruiting & NCT03299946 \\
\hline Cabozantinib & Nivolumab & । & 18 & $\begin{array}{l}\text { Advanced Solid Tumors in Patients } \\
\text { with HIV Infection }\end{array}$ & Recruiting & NCT04514484 \\
\hline Cabozantinib & Pembrolizumab & $\|$ & 29 & Liver cancer not eligible for local therapy & Recruiting & NCT04442581 \\
\hline Sorafenib/lenvatinib & Atezolizumab & III & 554 & $\begin{array}{l}\text { Locally advanced or metastatic and/or } \\
\text { unresectable HCC participants following } \\
\text { prior HCC treatment with atezolizumab } \\
\text { and bevacizumab combination }\end{array}$ & Not yet recruiting & NCT04770896 \\
\hline Bevacizumab & Nivolumab & $\|$ & 60 & Advanced HCC & Recruiting & NCT04393220 \\
\hline Bevacizumab & Atezolizumab & III & 662 & $\begin{array}{l}\text { HCC with high risk of recurrence after } \\
\text { surgical resection or ablation }\end{array}$ & Recruiting & NCT04102098 \\
\hline Bevacizumab & Atezolizumab & III & 150 & $\begin{array}{l}\text { Unresectable HCC without prior } \\
\text { systemic treatment }\end{array}$ & Recruiting & NCT04487067 \\
\hline Bevacizumab & Atezolizumab & III & 480 & $\begin{array}{l}\text { Locally advanced or metastatic HCC } \\
\text { without prior systemic treatment }\end{array}$ & Completed & NCT03434379 \\
\hline
\end{tabular}

the combination of multiple ICIs for tumour treatment may result in enhanced efficacy (Table 3) [106]. The inhibition of PD-1/PD-L1 can activate prospective tumour immunity only if there are enough CD8+ T cells in the tumour tissue. Moreover, anti-CTLA-4 antibodies can increase the abundance of active CD8+ T cells by inhibiting the B7-CTLA-4 pathway and then promoting the infiltration of active CD8+ $\mathrm{T}$ cells into tumour tissue, thus enhancing the antitumour effect [107]. This principle provides the rationale for ongoing clinical trials of the simultaneous blockade of multiple immune checkpoints. The CheckMate 040 trial tested the effects of nivolumab plus ipilimumab in the second-line setting for advanced HCC patients [108]. Nivolumab in combination with ipilimumab had acceptable safety, an optimistic object response rate (ORR), and a durable response in that randomized trial. Another phase I/II trial to evaluate the efficacy and safety of durvalumab plus tremelimumab in unresectable HCC has also been reported [109]. The results of the experiment are exciting, and the next phase of the study should prove worthwhile. Finally, a promising direction of development is to establish more relevant preclinical models of HCC to identify the most potent combination strategies between anti-
PD-1/PD-L1 antibodies and alternative immunomodulatory agents for further clinical development. There are various ongoing combination therapies of different ICIs in HCC (Table 3).

Combination therapies are currently in diverse phases of clinical exploitation to optimize antitumour efficacy. The combination of locoregional therapy with immunotherapy and targeted therapy for advanced HCC plays a huge role in the clinic, particularly for advanced HCC $[110,111]$.

\section{Currently approved treatment options}

Systemic therapy has been recognized as a substantial benefit for patients with HCC. Table 4 summarizes the systemic therapeutic agents for HCC that are approved by the FDA.

\section{First-line therapy Sorafenib}

Until 2017, sorafenib was the only potent treatment for patients who entered an advanced stage of HCC or failed other therapies. It is an oral MKI that blocks the activities of receptor tyrosine kinases (VEGFR-2/3, PDGFR- $\beta$, c-Kit, FLT-3, and RET), downstream pathway kinases 
Table 3 Ongoing trials investigating the combination therapy of $\mathrm{ICls}$

\begin{tabular}{|c|c|c|c|c|c|}
\hline Checkpoint inhibitors & Phase & Patients (No.) & Clinical setting & Status & NCT numbers \\
\hline Nivolumab + Ipilimumab & $|/| \mid$ & 32 & Child-Pugh A HCC & Recruiting & NCT03682276 \\
\hline Nivolumab + Ipilimumab & । & 50 & Unresectable HCC with Child Pugh Class A cirrhosis & Active, not recruiting & NCT03203304 \\
\hline Nivolumab + Ipilimumab & $\|$ & 30 & HCC with potential surgical resection & Active, not recruiting & NCT03222076 \\
\hline Nivolumab + Ipilimumab & III & 650 & Advanced HCC without prior systemic therapy & Recruiting & NCT04039607 \\
\hline Nivolumab + Ipilimumab & $\|$ & 40 & HCC with potential for curative surgical resection & Recruiting & NCT03510871 \\
\hline Nivolumab + Mogamulizumab & । & 96 & locally advanced or metastatic solid tumors & Completed & NCT02476123 \\
\hline Nivolumab + Relatlimab & । & 20 & Potentially resectable HCC & Not yet recruiting & NCT04658147 \\
\hline Pembrolizumab + Bavituximab & $\|$ & 28 & $\begin{array}{l}\text { Locally advanced or metastatic HCC not amenable } \\
\text { to locoregional therapy }\end{array}$ & Recruiting & NCT03519997 \\
\hline Durvalumab+ Tremelimumab & III & 1504 & Unresectable HCC without prior systemic therapy & Recruiting & NCT03298451 \\
\hline Durvalumab+ Tremelimumab & । & 32 & $\begin{array}{l}\text { Unresectable, locally advanced liver cancer after } \\
\text { radioembolization }\end{array}$ & Suspended & NCT04605731 \\
\hline Durvalumab+ Tremelimumab & $\|$ & 433 & Advanced HCC & Active, not recruiting & NCT02519348 \\
\hline
\end{tabular}

(Ras/Raf/MAPK and JAK/STAT), and other targets (BRaf and c- Raf) [112]. Sorafenib extends the mOS from 7.9 months to 10.7 months compared with the placebo arm [113]. The trial provides a standard study scheme for evaluating promising drugs. Similar benefits were identified in a parallel phase III trial in an Asian population, mostly with HBV-infected patients [114]. In later clinical trials, the OS of sorafenib-experienced patients appears to have increased; for example, the OS of patients in Asia increased from 6.5 months to 11 months and that of patients in the western region increased from 10.7 months to 15.1 months [115]. In these trials, the
AEs were mostly similar and manageable, including hand-foot skin reactions, rash or desquamation, hypertension, diarrhoea, fatigue, and ascites.

The success of sorafenib to treat advanced HCC seems beneficial for earlier clinical stages, but it did not obtain the expected benefit in the placebo-controlled clinical trial $[116,117]$. Although the widespread use of sorafenib increases the survival time of patients with HCC, its effective rate is low due to the formation of drug resistance [118]. Subsequently, several targeted drugs such as sunitinib, brivanib, erlotinib, linifanib, and lapatinib have been tested in a phase III trial over the past decade, but

Table 4 Systemic therapies approved for HCC by FDA

\begin{tabular}{|c|c|c|}
\hline Agent & Mechanism & FDA-approved indications \\
\hline \multicolumn{3}{|l|}{ First-line } \\
\hline Sorafenib & $\begin{array}{l}\text { Targeting VEGFR, PDGFR, C-KIT, RET, and } \\
\text { Ras/Raf/MEKJERK }\end{array}$ & $\begin{array}{l}\text { As monotherapy in patients with unresectable or metastasis } \\
\text { HCC }\end{array}$ \\
\hline Lenvatinib & Targeting VEGFR, PDGFR, FGFR, RET, and SCFR & As monotherapy in patients with unresectable HCC \\
\hline $\begin{array}{l}\text { Atezolizumab }+ \\
\text { Bevacizumab }\end{array}$ & Anti-PD-L1 antibody + Anti-VEGF antibody & $\begin{array}{l}\text { For patients with unresectable or metastatic HCC who have } \\
\text { not received prior systemic therapy }\end{array}$ \\
\hline \multicolumn{3}{|l|}{ Second-line } \\
\hline Regorafenib & $\begin{array}{l}\text { Targeting VEGFR, PDGFR, BRAF, FGFR, KIT, and } \\
\text { RET }\end{array}$ & As monotherapy in sorafenib-experienced patients \\
\hline Cabozantinib & Targeting VEGFR2, c-MET, AXL, KIT, and RET & As monotherapy in sorafenib-experienced patients \\
\hline Ramucirumab & Anti-VEGFR antibody & $\begin{array}{l}\text { As monotherapy in sorafenib-experienced patients with AFP } \\
\text { of } 400 \mathrm{ng} / \mathrm{ml} \text { or higher }\end{array}$ \\
\hline Nivolumab & Anti-PD-1 antibody & As monotherapy in sorafenib-experienced patients \\
\hline $\begin{array}{l}\text { Pembrolizumab } \\
\text { Nivolumab + Ipilimumab }\end{array}$ & $\begin{array}{l}\text { Anti-PD-1 antibody } \\
\text { Anti-PD-1 antibody + Anti-CTLA-4 antibody }\end{array}$ & $\begin{array}{l}\text { As monotherapy in sorafenib-experienced patients } \\
\text { As combination therapy in sorafenib-experienced patients }\end{array}$ \\
\hline
\end{tabular}


further breakthroughs were not made until the emergence of two other oral tyrosinase inhibitors: lenvatinib and regorafenib.

\section{Lenvatinib}

Lenvatinib is an oral MKI that blocks the behaviours of VEGFR1-3, FGFR1-4, PDGFR, RET, and KIT. In a phase III noninferiority randomized controlled trial involving 954 unresectable HCC patients worldwide, the role of lenvatinib was compared with sorafenib as a firstline therapy [119]. In this study, the mOS as the primary endpoint in the lenvatinib and sorafenib groups was 13.6 months and 12.3 months, respectively. With the tumour evaluation results conducted by mRECIST, lenvatinib had a statistically significant improvement in all secondary endpoints compared with sorafenib. Progression-free survival (PFS) and ORRs were significantly higher in the lenvatinib group (PFS: 7.4 months vs. 3.7 months; ORRs: $24.1 \%$ vs. $9.2 \%$ ). Moreover, lenvatinib had greater survival benefits for HBV-related HCC patients [120]. Additionally, lenvatinib plays a promising role in delaying the deterioration of diseases, including role function, pain, and diarrhoea [121].

Notably, the treatment-related AEs were similar in patients receiving lenvatinib or sorafenib, although accompanied by a different incidence, predominantly of hypertension and thrombocytopenia. This may be because the median duration of the treatment was longer in the lenvatinib group than in the sorafenib group [119].

These trial results indicated that lenvatinib is not inferior to sorafenib as a first-line setting and is regarded as an optimal candidate for sorafenib. Consequently, lenvatinib as a first-line treatment was recently supported by the ESMO, EASL, and ASCO guidelines [122-124].

\section{Atezolizumab plus bevacizumab}

Recently, reliable data on the combination of atezolizumab and bevacizumab have been presented, and this therapeutic approach is approved by the FDA and is used in patients with unresectable or metastatic HCC who have not received prior systemic treatment [125]. The combination of angiogenesis inhibitors and PD-L1 inhibitors is designed to inhibit high levels of angiogenesis and overactivity of VEGF and PD-L1 in HCC tumours. In an initial study, atezolizumab plus bevacizumab was efficacious and clinically meaningful, and it had obvious clinical benefits compared with atezolizumab alone [126]. Furthermore, this combination therapy was compared with sorafenib in the phase III IMbrave150 trial. The OS rates at 12 months of the combination group and the sorafenib group were 67.2 and $54.6 \%$, respectively. The combination treatment reduced the risk of death by $42 \%$ (HR: $0.58, P=0.0006$ ).
The mPFS was 6.8 months and 4.3 months in the respective groups, and the risk of disease progression or death was reduced by $41 \%$ (HR: $0.59, P<0.0001$ ) [102]. OS and PFS, as the primary endpoints of the study, met the predefined statistical threshold. Atezolizumab plus bevacizumab compared with sorafenib delayed the deterioration of quality of life (time to deterioration: 11.2 months vs. 3.6 months, respectively). In addition, the grade 3-4 side effects of combination treatment were mainly hypertension and proteinuria, with no difference from those of atezolizumab and bevacizumab alone. In Chinese patients with advanced HCC, combination therapy has also resulted in substantial clinical improvements in OS and PFS [127].

Atezolizumab plus bevacizumab to treat HCC was first included in the ASCO guidelines in 2020 on the basis of the IMbrave150 trial [124].

\section{Subsequent therapy}

The efficacy of first-line agents in patients with HCC may be restricted by contraindications, drug resistance, and AEs, and it is necessary to replace first-line agents with sequential treatment in these situations.

\section{Regorafenib}

Following the successful inclusion of regorafenib in the second-line setting, sequential therapy has been recognized as the standard modality. Regorafenib has profound antiproliferative and antiangiogenic effects on tumour cells by inhibiting VEGFR signal transduction, thereby regulating the tumour microenvironment. Its chemical structure differs from sorafenib only by a fluorine-carbon atom on the central benzene ring [128]. A randomized controlled phase III trial indicated mOS and mPFS improvement in further treatment of advanced HCC in sorafenib-experienced patients (mOS: 10.6 months vs 7.8 months; mPFS: 3.1 months vs 1.5 months). Meanwhile, the ORR, time to progression, and disease control rate (DCR) were also been increased. The AEs in the regorafenib group were similar to those in the sorafenib group, including high blood pressure, hand and foot skin reactions, fatigue, and diarrhoea [129]. Encouragingly, the study results of sorafenib-regorafenib sequential therapy demonstrated that from sorafenib treatment to death, the mOS was 26.0 months in the regorafenib group and 19.2 months in the placebo group [130]. There is evidence that the response rate to regorafenib is correlated with HCC molecular subtypes [131]. Therefore, regorafenib can bring significant survival benefits and can be used as an important choice for second-line therapy.

\section{Cabozantinib}

Cabozantinib is an oral MKI of VEGFR2, MET, AXL, $\mathrm{KIT}$, and RET. In contrast to regorafenib, cabozantinib 
has been mainly developed as a second-line therapy for Child-Pugh A HCC. A phase 3 study of cabozantinib in the second-line setting for patients with advanced HCC showed improvement in OS, mPFS, and DCR compared with a placebo [132]. Cabozantinib increased the mPFS to 5.2 months from 1.9 months in the placebo group, and the risk of death in the cabozantinib group was reduced by $24 \%$. The ORR was also significantly improved by $4.0 \%$ in the cabozantinib group and $0.4 \%$ in the placebo group. The majority of AEs were hand-foot syndrome, hypertension, fatigue, and transaminase elevation. The efficacy of cabozantinib is not completely consistent with the liver function of patients, but it shows a trend of a higher curative effect in patients with better liver function [133].

\section{Ramucirumab}

Briefly, ramucirumab is an anti-VEGFR2 $\mathrm{mAb}$ that directly targets the extracellular domain of VEGFR2 and prevents ligand-independent VEGFR2 signalling [134]. Compared with a placebo, ramucirumab did not greatly increase the survival time of unselected $\mathrm{HCC}$ patients [135]. However, ramucirumab increased the OS of advanced $\mathrm{HCC}$ patients with AFP $>400 \mathrm{ng} / \mathrm{ml}$ from 7.3 months to 8.5 months [136]. In addition, from 2015 to 2017, testing was conducted on 292 patients who still had tumour progression after receiving sorafenib. The primary inclusion criteria were impaired liver function, an AFP level of $400 \mathrm{ng} / \mathrm{mL}$ or greater, and resistance to sorafenib or progressive disease under sorafenib. The mOS and PFS were significantly increased in this specific group with approximately the same conclusions as previously [137]. Therefore, ramucirumab is a feasible second-line drug in advanced HCC patients with high AFP levels.

\section{Nivolumab}

Nivolumab is the first anti-PD-1 antibody approved for the treatment of patients with HCC. In the CheckMate040 study, the manageable safety and durable efficacy of nivolumab treatment were indicated in ITT and Asian patients previously treated with sorafenib. The study results show an ORR of $20 \%$ and a DCR of $60 \%$, and the efficacy is long lasting [138]. Unfortunately, as a first-line treatment for advanced HCC in CheckMate459, the primary endpoint of the nivolumab group (OS) was not statistically significant compared with sorafenib [68]. Following the results of the CheckMate040 study, nivolumab was conditionally granted approval by the FDA for the second-line treatment of HCC.

\section{Pembrolizumab}

Pembrolizumab, another antibody against PD-1, is commonly considered a second-line treatment option for
HCC. This decision is based on a nonrandomized KEYNOTE-224 study [81]. In this trial, a mOS of 12.9 months, a mPFS of 4.9 months, an ORR of $17 \%$, and a DCR of $61 \%$ were recorded. The results of the subsequent randomized phase III trial have also been reported. The study results show that the preset statistically significant endpoint was not reached; however, the ORR of the pembrolizumab group was much higher than that of the placebo group (18.3\% vs. $4.4 \%)$ [139]. Additionally, Asian people who received pembrolizumab had greater benefits with regard to OS, with an HR of 0.548 (95\% CI $0.374,0.804, P=0.0009$ ), and the survival benefit was better than that of patients in western regions [140].

\section{Nivolumab plus ipilimumab}

Recently, nivolumab plus ipilimumab was authorized by the FDA as a second-line treatment option for patients with advanced HCC who have received sorafenib. This novel treatment regimen achieved the combination blockade of the immune checkpoints PD-1 and CTLA-4, thereby improving the therapeutic effect for advanced HCC [141]. In the CheckMate-040 study, the efficacy and safety of the combination therapy with nivolumab and ipilimumab were assessed. This combination therapy appears to be safe and tolerable with a confirmed ORR of 33\%. As another main efficacy outcome, the duration of response ranged from 4.6 months to $30.5+$ months, with $31 \%$ of responses lasting at least 24 months. Moreover, combination therapy has shown common AEs similar to those seen with single-drug use. Subsequently, there have been additional clinical studies to evaluate the efficacy of combination immune checkpoint blockade for advanced HCC.

\section{Challenges and future directions}

There are several issues that need to be addressed soon with regard to the treatment of HCC (Fig. 3). Specifically, some biomarkers may be instructive for patient sensitivity to drugs and prognostic implications, such as AFP, which successfully influences treatment decisions in HCC [136]; no additional biomarkers have yet fulfilled this function. While extensive studies have been conducted to examine potential biomarkers to manage HCC [142-145], no available regimen has been developed to treat this tumour. This situation leads to the additional point that the optimal sequence strategy for these new agents is not yet clear. Therefore, it is critical to develop drug sensitivity biomarkers and prognostic biomarkers that identify patients who respond to appropriate treatments, to adopt a novel treatment strategy. Systematic and standardized collection and analysis of blood samples and tumour tissues from patients with HCC should 


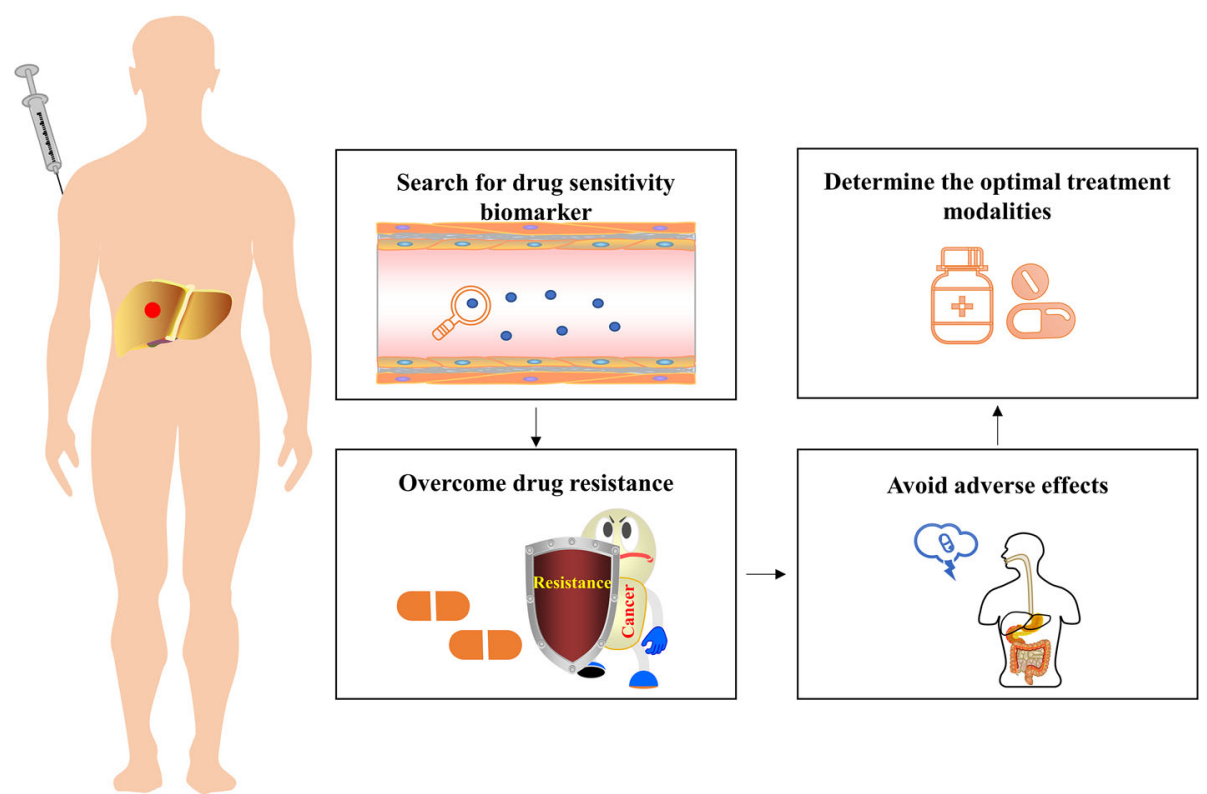

Fig. 3 Future directions in targeted therapy for HCC

be performed to further develop biomarker-based research.

In the clinic, drug resistance is also a major obstacle to systemic therapy for the treatment of HCC, particularly when newly developed drugs for HCC do not have remarkable results similar to those of other malignancies. The known mechanisms associated with resistance to targeted therapy include epigenetic biological processes, transport processes, regulated cell death, and the tumour microenvironment $[146,147]$. Drug resistance, i.e., primary resistance and acquired resistance, is still the main reason for treatment failure during targeted therapy [148]. In addition, the AEs caused by the drugs themselves can have undesirable impacts on the healthrelated quality of life for patients, even leading to drug discontinuation [149]. These conditions can also be alleviated with combination and sequential therapies.

Finally, it is worth considering how to establish relevant preclinical models based on the heterogeneity of HCC and the tumour microenvironment and then integrate them with data collected from the human body.

\section{Conclusions}

Systemic treatment for patients with HCC is changing due to ever-improving molecular targeted therapies and immunotherapies. The evolution of targeted therapies provides rich data and promising results, particularly in patients who are not amenable to locoregional treatments.

The key success lies in tyrosine kinase inhibitors, which remarkably increase overall survival as a systemic therapy. There is hope for treating HCC at an early or intermediate stage with a better understanding of the molecular mechanisms of hepatocarcinogenesis or the discovery of potential biomarkers. Targeted and immune-based therapies will gradually become the standard treatment for HCC, offering a new strategy for advanced HCC treatment and management. Angiogenesis inhibitors and immune checkpoint inhibitors remain the mainstream approaches to treat HCC. Of these, the research results of several targeted agents in recent years have been successful.

Various clinical trials of systemic therapies are ongoing and have proven successful thus far. These trials are useful in determining the role and effect of molecular targeted therapies and immunotherapies, defining the optimal combinations, and assessing the optimal timing, i.e., adjuvant therapy and first-line or second-line therapy, and thus establishing rational treatment principles. Further exploration of targeted and immunotherapies in $\mathrm{HCC}$ is of great practical importance for controlling disease progression, improving the survival quality of patients, and guiding the rational use of clinical drugs.

\section{Abbreviations}

AE: adverse event; AFP: alpha fetoprotein; ASCO: American Society of Clinical Oncology; BCLC: Barcelona Clinic Liver Cancer; Cl: confidence interval.; CIK: cytokine-induced killer; DCR: disease control rate; EGFR: epidermal growth factor; FDA: Food and Drug Administration; FGF: fibroblast growth factor; HBV: hepatitis B virus; HCC: hepatocellular carcinoma; HCV: hepatitis C virus; HR: hazard ratio.; ICIs: immune checkpoint inhibitors; IGFR: insulin-like growth factor receptor; JAKJSTAT: Janus kinase/signal transducer activator of transcription factor pathway; LAG-3: lymphocyte activation gene-3; mAb: monoclonal antibodies; mOS: median overall survival; mPFS: median progression-free survival; ncRNA: noncoding RNA; MKI: multikinase inhibitor; NA: not available; NE: not evaluated; ORR: object response rate; OS: overall survival; PDGF: platelet-derived growth factor; PD-1: programmed death-1; 
PD-L1: programmed death ligand-1; PFS: progression-free survival; PI3K-AKTmTOR: phosphoinositide 3-kinase-AKT-mammalian target of rapamycin pathway; PR: partial response; PTEN: phosphate and tension homology deleted on chromosome ten; Ras-Raf-MAPK: mitogen-activated protein kinase pathway; RECIST: response evaluation criteria in solid tumours; RFS: relapse-free survival.; TGF- $\beta$ : transforming growth factor- $\beta$; TIGIT: T cell lg and immunoreceptor tyrosine-based inhibitory motif domain; TIM-3: T cell immunoglobulin and mucin-containing protein 3; TKI: tyrosine kinase inhibitor; TTP: time to progression; VEGF: vascular endothelial growth factor; VISTA: $V$-domain Ig suppressor of $\mathrm{T}$ cell activation.

\section{Acknowledgements}

Not applicable.

\section{Authors' contributions}

All authors made substantial and intellectual contributions to the review. Under the direction of the corresponding author, Xin Qing and Wenjing $\mathrm{Xu}, \mathrm{organized}$ and wrote this review, and Jingjing Zong, Xuanlong Du, and Hao Peng commented on this manuscript. The author(s) read and approved the final manuscript

\section{Funding}

This study was supported by grants from the National Natural Science Foundation of China (Nos. 81872255 and 62041101) and the Key Medical Talents Foundation of Jiangsu Province (2016KJQWZDRC-03).

\section{Availability of data and materials}

Not applicable.

\section{Declarations}

Ethics approval and consent to participate

Not applicable.

\section{Consent for publication}

All authors consent to publication.

\section{Competing interests}

The authors declare no competing interests.

\section{Received: 22 May 2021 Accepted: 5 August 2021}

\section{Published online: 21 August 2021}

\section{References}

1. Bray F, Ferlay J, Soerjomataram I, Siegel RL, Torre LA, Jemal A. Global cancer statistics 2018: GLOBOCAN estimates of incidence and mortality worldwide for 36 cancers in 185 countries. CA Cancer J Clin. 2018;68:394-424. https:// doi.org/10.3322/caac.21492.

2. Villanueva A. Hepatocellular Carcinoma. N Engl J Med. 2019;380:1450-62. https://doi.org/10.1056/NEJMra1713263.

3. Vitale A, Peck-Radosavljevic M, Giannini EG, Vibert E, Sieghart W, van Poucke S, et al. Personalized treatment of patients with very early hepatocellular carcinoma. J Hepatol. 2017;66(2):412-23. https://doi.org/10.1016/j.jhep.2016. 09.012.

4. Kulik L, El-Serag HB. Epidemiology and Management of Hepatocellular Carcinoma. Gastroenterology. 2019;156:477-491.e1. https://doi.org/10.1053/j. gastro.2018.08.065

5. Deng G-L, Zeng S, Shen H. Chemotherapy and target therapy for hepatocellular carcinoma: new advances and challenges. World J Hepatol. 2015;7:787-98. https://doi.org/10.4254/wjh.v7.i5.787.

6. Kudo M. Management of Hepatocellular Carcinoma in Japan as a worldleading model. Liver Cancer. 2018;7(2):134-47. https://doi.org/10.1159/0004 84619.

7. Drosten M, Barbacid M. Targeting the MAPK pathway in KRAS-driven tumors. Cancer Cell. 2020;37:543-50. https://doi.org/10.1016/j.ccell.2020.03. 013.

8. Porta C, Paglino C, Mosca A. Targeting PI3K/Akt/mTOR signaling in Cancer. Front Oncol. 2014;4:64. https://doi.org/10.3389/fonc.2014.00064.

9. Lokau J, Schoeder V, Haybaeck J, Garbers C. Jak-Stat signaling induced by Interleukin-6 family cytokines in hepatocellular carcinoma. Cancers (Basel). 2019;11(11):1704. https://doi.org/10.3390/cancers11111704.
10. Dimri M, Satyanarayana A. Molecular signaling pathways and therapeutic targets in hepatocellular carcinoma. Cancers (Basel). 2020;12(2). https://doi. org/10.3390/cancers12020491.

11. Dhanasekaran R, Nault J-C, Roberts LR, Zucman-Rossi J. Genomic medicine and implications for hepatocellular carcinoma prevention and therapy. Gastroenterology. 2019;156:492-509. https://doi.org/10.1053/.gastro.2018.11.001.

12. Cheng H, Sun G, Chen H, Li Y, Han Z, Li Y, et al. Trends in the treatment of advanced hepatocellular carcinoma: immune checkpoint blockade immunotherapy and related combination therapies. Am J Cancer Res. 2019; 9:1536-45.

13. Abd El Aziz MA, Facciorusso a, Nayfeh T, Saadi S, Elnaggar M, Cotsoglou C, Sacco R. Immune Checkpoint Inhibitors for Unresectable Hepatocellular Carcinoma. Vaccines (Basel) 2020;8(4):616. https://doi.org/10.3390/va ccines8040616

14. Pinter M, Peck-Radosavljevic M. Review article: systemic treatment of hepatocellular carcinoma. Aliment Pharmacol Ther. 2018;48:598-609. https:// doi.org/10.1111/apt.14913.

15. Huang A, Yang X-R, Chung W-Y, Dennison AR, Zhou J. Targeted therapy for hepatocellular carcinoma. Signal Transduct Target Ther. 2020;5:146. https:// doi.org/10.1038/s41392-020-00264-X.

16. Prieto J, Melero I, Sangro B. Immunological landscape and immunotherapy of hepatocellular carcinoma. Nat Rev Gastroenterol Hepatol. 2015;12:681700. https://doi.org/10.1038/nrgastro.2015.173.

17. Vogel A, Saborowski A. Current strategies for the treatment of intermediate and advanced hepatocellular carcinoma. Cancer Treat Rev. 2020;82:101946. https://doi.org/10.1016/j.ctrv.2019.101946.

18. Chen S, Cao Q, Wen W, Wang H. Targeted therapy for hepatocellular carcinoma: challenges and opportunities. Cancer Lett. 2019;460:1-9. https:// doi.org/10.1016/j.canlet.2019.114428.

19. Chen C, Wang G. Mechanisms of hepatocellular carcinoma and challenges and opportunities for molecular targeted therapy. World J Hepatol. 2015;7: 1964-70. https://doi.org/10.4254/wjh.v7.i15.1964.

20. Ghouri YA, Mian I, Rowe JH. Review of hepatocellular carcinoma: epidemiology, etiology, and carcinogenesis. J Carcinog 2017;16:1. https:// doi.org/10.4103/jcar.JCar_9_16.

21. Yuan J, Dong X, Yap J, Hu J. The MAPK and AMPK signalings: interplay and implication in targeted cancer therapy. J Hematol Oncol. 2020;13(1):113. https://doi.org/10.1186/s13045-020-00949-4

22. Huynh H, Nguyen TT, Chow K-HP, Tan PH, Soo KC, Tran E. Over-expression of the mitogen-activated protein kinase (MAPK) kinase (MEK)-MAPK in hepatocellular carcinoma: its role in tumor progression and apoptosis. BMC Gastroenterol. 2003;3:19. https://doi.org/10.1186/1471-230X-3-19.

23. Tsuboi $Y$, Ichida T, Sugitani S, Genda T, Inayoshi J, Takamura M, et al. Overexpression of extracellular signal-regulated protein kinase and its correlation with proliferation in human hepatocellular carcinoma. Liver Int. 2004;24:432-6. https://doi.org/10.1111/j.1478-3231.2004.0940.x.

24. Hwang YH, Choi JY, Kim S, Chung ES, Kim T, Koh SS, et al. Over-expression of c-raf-1 proto-oncogene in liver cirrhosis and hepatocellular carcinoma. Hepatol Res. 2004;29:113-21. https://doi.org/10.1016/j.hepres.2004.02.009.

25. Min L, He B, Hui L. Mitogen-activated protein kinases in hepatocellular carcinoma development. Semin Cancer Biol. 2011;21:10-20. https://doi.org/1 0.1016/j.semcancer.2010.10.011.

26. Chen $L$, Shi $Y$, Jiang $C-Y$, Wei $L-X$, Wang $Y-L$, Dai G-H. Expression and prognostic role of pan-Ras, Raf-1, pMEK1 and pERK1/2 in patients with hepatocellular carcinoma. Eur J Surg Oncol. 2011;37:513-20. https://doi. org/10.1016/j.ejso.2011.01.023.

27. Zhou X, Zhu A, Gu X, Xie G. Inhibition of MEK suppresses hepatocellular carcinoma growth through independent MYC and BIM regulation. Cell Oncol (Dordr). 2019:42:369-80. https://doi.org/10.1007/s13402-019-00432-4.

28. Lim HY, Merle P, Weiss KH, Yau T, Ross P, Mazzaferro V, et al. Phase II studies with Refametinib or Refametinib plus Sorafenib in patients with RASmutated hepatocellular carcinoma. Clin Cancer Res. 2018;24:4650-61. https://doi.org/10.1158/1078-0432.CCR-17-3588.

29. Kim R, Tan E, Wang E, Mahipal A, Chen D-T, Cao B, et al. A phase I trial of Trametinib in combination with Sorafenib in patients with advanced hepatocellular Cancer. Oncologist. 2020;25(12):e1893-1899. https://doi.org/1 0.1634/theoncologist.2020-0759.

30. Chung T-W, Lee Y-C, Kim C-H. Hepatitis B viral HBx induces matrix metalloproteinase-9 gene expression through activation of ERK and PI-3K AKT pathways: involvement of invasive potential. FASEB J. 2004;18:1123-5. https://doi.org/10.1096/fj.03-1429fje. 
31. Erhardt A, Hassan M, Heintges T, Häussinger D. Hepatitis C virus core protein induces cell proliferation and activates ERK, JNK, and p38 MAP kinases together with the MAP kinase phosphatase MKP-1 in a HepG2 Tet-off cell line. Virology. 2002;292:272-84. https://doi.org/10.1006/viro.2 001.1227.

32. Mossmann D, Park S, Hall MN. mTOR signalling and cellular metabolism are mutual determinants in cancer. Nat Rev Cancer. 2018;18:744-57. https://doi. org/10.1038/s41568-018-0074-8.

33. Ferrín G, Guerrero M, Amado V, Rodríguez-Perálvarez M, de LMM. Activation of mTOR Signaling Pathway in Hepatocellular Carcinoma. Int J Mol Sci. 2020;21(4):1266. https://doi.org/10.3390/ijms21041266.

34. Khalid A, Hussain T, Manzoor S, Saalim M, Khaliq S. PTEN: a potential prognostic marker in virus-induced hepatocellular carcinoma. Tumour Biol. 2017;39:1010428317705754. https://doi.org/10.1177/1010428317705754.

35. Matter MS, Decaens T, Andersen JB, Thorgeirsson SS. Targeting the mTOR pathway in hepatocellular carcinoma: current state and future trends. J Hepatol. 2013;60:855-65. https://doi.org/10.1016/j.jhep.2013.11.031.

36. Zhou L, Huang Y, Li J, Wang Z. The mTOR pathway is associated with the poor prognosis of human hepatocellular carcinoma. Med Oncol. 2010;27: 255-61. https://doi.org/10.1007/s12032-009-9201-4.

37. Sahin F, Kannangai R, Adegbola O, Wang J, Su G, Torbenson M. mTOR and P70 S6 kinase expression in primary liver neoplasms. Clin Cancer Res. 2004; 10(24):8421-5. https://doi.org/10.1158/1078-0432.CCR-04-0941.

38. Andersen JB, Spee B, Blechacz BR, Avital I, Komuta M, Barbour A, et al. Genomic and genetic characterization of cholangiocarcinoma identifies therapeutic targets for tyrosine kinase inhibitors. Gastroenterology. 2012;142: 1021-1031.e15. https://doi.org/10.1053/j.gastro.2011.12.005

39. Lu X, Paliogiannis $P$, Calvisi DF, Chen $X$. Role of the mammalian target of rapamycin pathway in liver Cancer: from molecular genetics to targeted therapies. Hepatology. 2021;73(Suppl 1):49-61. https://doi.org/10.1002/ hep.31310.

40. Yang S, Liu G. Targeting the Ras/Raf/MEK/ERK pathway in hepatocellular carcinoma. Oncol Lett. 2017;13(3):1041-7. https://doi.org/10.3892/ol.2017.5557.

41. Han T, Xiang D-M, Sun W, Liu N, Sun H-L, Wen W, et al. PTPN11/Shp2 overexpression enhances liver cancer progression and predicts poor prognosis of patients. J Hepatol. 2015;63:651-60. https://doi.org/10.1016/j. jhep.2015.03.036

42. Zhu Y-J, Zheng B, Wang H-Y, Chen L. New knowledge of the mechanisms of sorafenib resistance in liver cancer. Acta Pharmacol Sin. 2017;38(5):61422. https://doi.org/10.1038/aps.2017.5.

43. Schnitzbauer AA, Filmann N, Adam R, Bachellier P, Bechstein WO, Becker T, et al. mTOR inhibition is Most beneficial after liver transplantation for hepatocellular carcinoma in patients with active tumors. Ann Surg. 2020; 272(5):855-62. https://doi.org/10.1097/SLA.0000000000004280.

44. Perugorria MJ, Olaizola P, Labiano I, Esparza-Baquer A, Marzioni M, Marin $J J G$, et al. Wnt- $\beta$-catenin signalling in liver development, health and disease. Nat Rev Gastroenterol Hepatol. 2019;16:121-36. https://doi.org/10.1038/s41 575-018-0075-9

45. Cui Y, Wu X, Lin C, Zhang X, Ye L, Ren L, et al. AKIP1 promotes early recurrence of hepatocellular carcinoma through activating the Wnt/ $\beta$ catenin/CBP signaling pathway. Oncogene. 2019;38:5516-29. https://doi. org/10.1038/s41388-019-0807-5

46. Vilchez V, Turcios L, Marti F, Gedaly R. Targeting Wnt/B-catenin pathway in hepatocellular carcinoma treatment. World J Gastroenterol. 2016;22:823-32. https://doi.org/10.3748/wjg.v22.i2.823.

47. Khalaf AM, Fuentes D, Morshid Al, Burke MR, Kaseb AO, Hassan M, et al. Role of Wnt/ $\beta$-catenin signaling in hepatocellular carcinoma, pathogenesis, and clinical significance. J Hepatocell Carcinoma. 2018;5:61-73. https://doi.org/1 $0.2147 / J H C . S 156701$

48. Bruix J, Sherman M. Management of hepatocellular carcinoma: an update. Hepatology. 2011;53:1020-2. https://doi.org/10.1002/hep.24199.

49. Hu Z, Wang $P$, Lin J, Zheng $X$, Yang F, Zhang G, et al. MicroRNA-197 promotes metastasis of hepatocellular carcinoma by activating Wnt/ $\beta$ catenin signaling. Cell Physiol Biochem. 2018;51:470-86. https://doi.org/1 $0.1159 / 000495242$

50. Srisuttee R, Koh SS, Kim SJ, Malilas W, Boonying W, Cho I-R, et al. Hepatitis B virus $X(H B X)$ protein upregulates $\beta$-catenin in a human hepatic cell line by sequestering SIRT1 deacetylase. Oncol Rep. 2012;28:276-82. https://doi.org/1 0.3892/or.2012.1798

51. Wang W, Pan Q, Fuhler GM, Smits R, Peppelenbosch MP. Action and function of Wnt/ $\beta$-catenin signaling in the progression from chronic hepatitis C to hepatocellular carcinoma. J Gastroenterol. 2017:52:419-31. https://doi.org/10.1007/s00535-016-1299-5.

52. Kan Z, Zheng H, Liu X, Li S, Barber TD, Gong Z, et al. Whole-genome sequencing identifies recurrent mutations in hepatocellular carcinoma. Genome Res. 2013;23:1422-33. https://doi.org/10.1101/gr.154492.113.

53. Hin Tang JJ, Hao Thng DK, Lim JJ, Toh TB. JAK/STAT signaling in hepatocellular carcinoma. Hepat Oncol. 2020;7:HEP18. https://doi.org/10.221 7/hep-2020-0001.

54. Yang S, Luo C, Gu Q, Xu Q, Wang G, Sun H, et al. Activating JAK1 mutation may predict the sensitivity of JAK-STAT inhibition in hepatocellular carcinoma. Oncotarget. 2016;7:5461-9. https://doi.org/10.18632/oncotarget. 6684.

55. Wang X, Liao X, Yu T, Gong Y, Zhang L, Huang J, et al. Analysis of clinical significance and prospective molecular mechanism of main elements of the JAK/STAT pathway in hepatocellular carcinoma. Int J Oncol. 2019;55:805-22. https://doi.org/10.3892/ijo.2019.4862.

56. Maugeri-Saccà M, de Maria R. The hippo pathway in normal development and cancer. Pharmacol Ther. 2018;186:60-72. https://doi.org/10.1016/j.pha rmthera.2017.12.011.

57. Calses PC, Crawford JJ, Lill JR, Dey A. Hippo pathway in Cancer: aberrant regulation and therapeutic opportunities. Trends Cancer. 2019;5:297-307. https://doi.org/10.1016/j.trecan.2019.04.001.

58. Perra A, Kowalik MA, Ghiso E, Ledda-Columbano GM, Di Tommaso L, Angioni MM, et al. YAP activation is an early event and a potential therapeutic target in liver cancer development. J Hepatol. 2014;61(5):108896. https://doi.org/10.1016/j.jhep.2014.06.033.

59. Zhang S, Zhou D. Role of the transcriptional coactivators YAP/TAZ in liver cancer. Curr Opin Cell Biol. 2019;61:64-71. https://doi.org/10.1016/j.ceb.2019. 07.006 .

60. Zhao B, Wei X, Li W, Udan RS, Yang Q, Kim J, et al. Inactivation of YAP oncoprotein by the hippo pathway is involved in cell contact inhibition and tissue growth control. Genes Dev. 2007;21:2747-61. https://doi.org/10.1101/ gad.1602907.

61. Reis H, Bertram S, Pott L, Canbay A, Gallinat A, Baba HA. Markers of hippopathway activity in tumor forming liver lesions. Pathol Oncol Res. 2017;23: 33-9. https://doi.org/10.1007/s12253-016-0079-0.

62. Morse MA, Sun W, Kim R, He AR, Abada PB, Mynderse M, et al. The role of angiogenesis in hepatocellular carcinoma. Clin Cancer Res. 2019;25:912-20. https://doi.org/10.1158/1078-0432.CCR-18-1254.

63. Zhang L, Wang J-N, Tang J-M, Kong $X$, Yang J-Y, Zheng F, et al. VEGF is essential for the growth and migration of human hepatocellular carcinoma cells. Mol Biol Rep. 2012;39:5085-93. https://doi.org/10.1007/s11033-011-13 04-2.

64. Dai S, Zhou Z, Chen Z, Xu G, Chen Y. Fibroblast growth factor receptors (FGFRs): structures and small molecule inhibitors. Cells. 2019;8(6):614. https:// doi.org/10.3390/cells8060614

65. Demoulin J-B, Essaghir A. PDGF receptor signaling networks in normal and cancer cells. Cytokine Growth Factor Rev. 2014;25:273-83. https://doi.org/1 0.1016/j.cytogfr.2014.03.003.

66. Du Z, Lovly CM. Mechanisms of receptor tyrosine kinase activation in cancer. Mol Cancer. 2018;17:58. https://doi.org/10.1186/s12943-018-0782-4.

67. Llovet JM, Ricci S, Mazzaferro V, Hilgard P, Gane E, Blanc J-F, et al. Sorafenib in advanced hepatocellular carcinoma. N Engl J Med. 2008;359:378-90. https://doi.org/10.1056/NEJMoa0708857.

68. Yau T, Park JW, Finn RS, Cheng A-L, Mathurin P, Edeline J, et al. CheckMate 459: a randomized, multi-center phase III study of nivolumab (NIVO) vs sorafenib (SOR) as first-line (1L) treatment in patients (pts) with advanced hepatocellular carcinoma (aHCC). Ann Oncol. 2019;30:v874-5. https://doi. org/10.1093/annonc/mdz394.029.

69. Qin S, Li A, Yi M, Yu S, Zhang M, Wu K. Recent advances on antiangiogenesis receptor tyrosine kinase inhibitors in cancer therapy. J Hematol Oncol. 2019;12:27. https://doi.org/10.1186/s13045-019-0718-5.

70. Neureiter D, Stintzing S, Kiesslich T, Ocker M. Hepatocellular carcinoma: therapeutic advances in signaling, epigenetic and immune targets. World J Gastroenterol. 2019;25:3136-50. https://doi.org/10.3748/wjg.v25.i25.3136.

71. Bangaru S, Marrero JA, Singal AG. Review article: new therapeutic interventions for advanced hepatocellular carcinoma. Aliment Pharmacol Ther. 2020;51(1):78-89. https://doi.org/10.1111/apt.15573.

72. Berretta M, Rinaldi L, Di Benedetto F, Lleshi A, De RV, Facchini G, et al. Angiogenesis Inhibitors for the Treatment of Hepatocellular Carcinoma. Front Pharmacol. 2016;7:428. https://doi.org/10.3389/fphar.2016.00428. 
73. Huang S-Z, Wei M-N, Huang J-R, Zhang Z-J, Zhang W-J, Jiang Q-W, et al. Targeting TF-AKT/ERK-EGFR pathway suppresses the growth of hepatocellular carcinoma. Front Oncol. 2019;9:150. https://doi.org/10.3389/ fonc.2019.00150.

74. Lim HY, Heo J, Choi HJ, Lin C-Y, Yoon J-H, Hsu C, et al. A phase II study of the efficacy and safety of the combination therapy of the MEK inhibitor refametinib (BAY 86-9766) plus sorafenib for Asian patients with unresectable hepatocellular carcinoma. Clin Cancer Res. 2014;20(23):597685. https://doi.org/10.1158/1078-0432.CCR-13-3445.

75. Tai WM, Yong WP, Lim C, Low LS, Tham CK, Koh TS, et al. A phase Ib study of selumetinib (AZD6244, ARRY-142886) in combination with sorafenib in advanced hepatocellular carcinoma (HCC). Ann Oncol. 2016;27:2210-5. https://doi.org/10.1093/annonc/mdw415.

76. Zhang J, Zong $Y, X u G-Z$, Xing K. Erlotinib for advanced hepatocellular carcinoma. A systematic review of phase II/III clinical trials. Saudi Med J. 2016;37:1184-90. https://doi.org/10.15537/smj.2016.11.16267.

77. Zongyi Y, Xiaowu L. Immunotherapy for hepatocellular carcinoma. Cance Lett. 2020;470:8-17. https://doi.org/10.1016/j.canlet.2019.12.002.

78. Cheng H-Y, Kang P-J, Chuang Y-H, Wang Y-H, Jan M-C, Wu C-F, et al. Circulating programmed death-1 as a marker for sustained high hepatitis B viral load and risk of hepatocellular carcinoma. PLoS One. 2014;9:e95870. https://doi.org/10.1371/journal.pone.0095870.

79. Ribas A, Wolchok JD. Cancer immunotherapy using checkpoint blockade. Science. 2018;359:1350-5. https://doi.org/10.1126/science.aar4060.

80. El-Khoueiry AB, Sangro B, Yau T, Crocenzi TS, Kudo M, Hsu C, et al. Nivolumab in patients with advanced hepatocellular carcinoma (CheckMate 040): an open-label, non-comparative, phase $1 / 2$ dose escalation and expansion trial. Lancet. 2017;389:2492-502. https://doi.org/10.1016/S01406736(17)31046-2.

81. Zhu AX, Finn RS, Edeline J, Cattan S, Ogasawara S, Palmer D, et al. Pembrolizumab in patients with advanced hepatocellular carcinoma previously treated with sorafenib (KEYNOTE-224): a non-randomised, openlabel phase 2 trial. Lancet Oncol. 2018;19(7):940-52. https://doi.org/10.1016/ S1470-2045(18)30351-6.

82. Qin S, Ren Z, Meng Z, Chen Z, Chai X, Xiong J, et al. Camrelizumab in patients with previously treated advanced hepatocellular carcinoma: a multicentre, open-label, parallel-group, randomised, phase 2 trial. Lancet Oncol. 2020;21:571-80. https://doi.org/10.1016/S1470-2045(20)30011-5.

83. Llovet JM, Montal R, Sia D, Finn RS. Molecular therapies and precision medicine for hepatocellular carcinoma. Nat Rev Clin Oncol. 2018;15:599616. https://doi.org/10.1038/s41571-018-0073-4.

84. Sangro B, Gomez-Martin C, La Mata M de, Iñarrairaegui M, Garralda E, Barrera P, et al. A clinical trial of CTLA-4 blockade with tremelimumab in patients with hepatocellular carcinoma and chronic hepatitis C. J Hepatol 2013;59:81-88. doi:https://doi.org/10.1016/j.jhep.2013.02.022.

85. Sharma P, Allison JP. The future of immune checkpoint therapy. Science. 2015;348(6230):56-61. https://doi.org/10.1126/science.aaa8172.

86. Matsui HM, Hazama S, Nakajima M, Xu M, Matsukuma S, Tokumitsu Y, et al. Novel adjuvant dendritic cell therapy with transfection of heat-shock protein 70 messenger RNA for patients with hepatocellular carcinoma: a phase I/II prospective randomized controlled clinical trial. Cancer Immunol Immunother. 2020;70(4):945-57. https://doi.org/10.1007/s00262-020-02737-y.

87. Rochigneux P, Chanez B, de Rauglaudre B, Mitry E, Chabannon C, Gilabert M. Adoptive cell therapy in hepatocellular carcinoma: biological rationale and first results in early phase Clinical trials. Cancers (Basel). 2021. https:// doi.org/10.3390/cancers13020271.

88. Shi D, Shi Y, Kaseb AO, Qi X, Zhang Y, Chi J, et al. Chimeric antigen receptor-Glypican-3 T-cell therapy for advanced hepatocellular carcinoma: results of phase I trials. Clin Cancer Res. 2020;26:3979-89. https://doi.org/1 0.1158/1078-0432.CCR-19-3259.

89. Zhao Z, Guo W, Fang S, Song S, Song J, Teng F, et al. An armored GPC3-directed CAR-T for refractory or relapsed hepatocellular carcinoma in China: a phase I trial. JCO. 2021;39:4095. https://doi.org/10.1200/JCO.2021.39.15_suppl.4095.

90. Li J, Xing J, Yang Y, Liu J, Wang W, Xia Y, et al. Adjuvant 1311-metuximab for hepatocellular carcinoma after liver resection: a randomised, controlled, multicentre, open-label, phase 2 trial. Lancet Gastroenterol Hepatol. 2020;5: 548-60. https://doi.org/10.1016/S2468-1253(19)30422-4.

91. Kaseb A, Duda DG, Tran Cao HS, Abugabal YI, Vence LM, Rashid A, et al. Randomized, open-label, perioperative phase II study evaluating nivolumab alone versus nivolumab plus ipilimumab in patients with resectable HCC. Ann Oncol. 2019;30:v880. https://doi.org/10.1093/annonc/mdz394.038.
92. Pinato DJ, Cortellini A, Pai M, Spalding D, Talbot T, Goldin RD, et al. PRIMEHCC: phase lb study of neoadjuvant ipilimumab and nivolumab prior to liver resection for hepatocellular carcinoma. JCO. 2021;39(15_suppl):e16131. https://doi.org/10.1200/JCO.2021.39.15 suppl.e16131.

93. Lee JH, Lee J-H, Lim Y-S, Yeon JE, Song T-J, Yu SJ, et al. Adjuvant immunotherapy with autologous cytokine-induced killer cells for hepatocellular carcinoma. Gastroenterology. 2015;148:1383-91.e6. https:// doi.org/10.1053/j.gastro.2015.02.055.

94. Yu SJ, Ma C, Heinrich B, Brown ZJ, Sandhu M, Zhang Q, et al. Targeting the crosstalk between cytokine-induced killer cells and myeloid-derived suppressor cells in hepatocellular carcinoma. J Hepatol. 2019;70:449-57. https://doi.org/10.1016/j.jhep.2018.10.040.

95. Kanda M, Sugimoto H, Kodera Y. Genetic and epigenetic aspects of initiation and progression of hepatocellular carcinoma. World J Gastroenterol. 2015;21(37):10584-97. https://doi.org/10.3748/wjg.v21.i37.1 0584

96. Wilson CL, Mann DA, Borthwick LA. Epigenetic reprogramming in liver fibrosis and cancer. Adv Drug Deliv Rev. 2017;121:124-32. https://doi.org/1 0.1016/j.addr.2017.10.011.

97. Bayo J, Fiore EJ, Dominguez LM, Real A, Malvicini M, Rizzo M, et al. A comprehensive study of epigenetic alterations in hepatocellular carcinoma identifies potential therapeutic targets. J Hepatol. 2019;71(1):78-90. https:// doi.org/10.1016/j.jhep.2019.03.007.

98. Khan FS, Ali I, Afridi UK, Ishtiaq M, Mehmood R. Epigenetic mechanisms regulating the development of hepatocellular carcinoma and their promise for therapeutics. Hepatol Int. 2017;11(1):45-53. https://doi.org/10.1007/s12 072-016-9743-4.

99. Anestopoulos I, Voulgaridou GP, Georgakilas AG, Franco R, Pappa A, Panayiotidis MI. Epigenetic therapy as a novel approach in hepatocellular carcinoma. Pharmacol Ther. 2015;145:103-19. https://doi.org/10.1016/j.pha rmthera.2014.09.005

100. Fukumura D, Kloepper J, Amoozgar Z, Duda DG, Jain RK. Enhancing cancer immunotherapy using antiangiogenics: opportunities and challenges. Nat Rev Clin Oncol. 2018;15:325-40. https://doi.org/10.1038/nrclinonc.2018.29.

101. Raybould AL, Sanoff H. Combination antiangiogenic and immunotherapy for advanced hepatocellular carcinoma: evidence to date. J Hepatocell Carcinoma. 2020;7:133-42. https://doi.org/10.2147/JHC.S224938.

102. Finn RS, Qin S, Ikeda M, Galle PR, Ducreux M, Kim T-Y, et al. Atezolizumab plus Bevacizumab in Unresectable Hepatocellular Carcinoma. N Engl J Med. 2020;382:1894-905. https://doi.org/10.1056/NEJMoa1915745.

103. Zhu AX, Finn RS, Ikeda $M$, Sung MW, Baron AD, Kudo $M$, et al. A phase lb study of lenvatinib (LEN) plus pembrolizumab (PEMBRO) in unresectable hepatocellular carcinoma (uHCC). JCO. 2020;38:4519. https://doi.org/10.1200/ JCO.2020.38.15_suppl.4519.

104. Bai L, Sun M, Xu A, Bai Y, Wu J, Shao G, et al. Phase 2 study of AK104 (PD-1/ CTLA-4 bispecific antibody) plus lenvatinib as first-line treatment of unresectable hepatocellular carcinoma. JCO. 2021;39:4101. https://doi.org/1 0.1200/JCO.2021.39.15_suppl.4101.

105. Xu J, Shen J, Gu S, Zhang Y, Wu L, Wu J, et al. Camrelizumab in combination with Apatinib in patients with advanced hepatocellular carcinoma (RESCUE): a nonrandomized, open-label, Phase II Trial. Clin Cancer Res. 2021;27:1003-11. https://doi.org/10.1158/1078-0432.CCR-20-2 571.

106. Cheng A-L, Hsu C, Chan SL, Choo S-P, Kudo M. Challenges of combination therapy with immune checkpoint inhibitors for hepatocellular carcinoma. J Hepatol. 2020;72(2):307-19. https://doi.org/10.1016/j.jhep.2019.09.025.

107. Kudo M. Combination Cancer immunotherapy in hepatocellular carcinoma. Liver Cancer. 2018;7:20-7. https://doi.org/10.1159/000486487.

108. Yau T, Kang Y-K, Kim T-Y, El-Khoueiry AB, Santoro A, Sangro B, et al. Efficacy and safety of Nivolumab plus Ipilimumab in patients with advanced hepatocellular carcinoma previously treated with Sorafenib: the CheckMate 040 randomized Clinical trial. JAMA Oncol. 2020;6(11):e204564. https://doi. org/10.1001/jamaoncol.2020.4564.

109. Kelley RK, Abou-Alfa GK, Bendell JC, Kim T-Y, Borad MJ, Yong W-P, et al. Phase $|/ /|$ study of durvalumab and tremelimumab in patients with unresectable hepatocellular carcinoma (HCC): phase I safety and efficacy analyses. JCO. 2017;35(15_suppl):4073. https://doi.org/10.1200/JCO.2017.35.1 5_suppl.4073.

110. Dendy MS, Ludwig JM, Stein SM, Kim HS. Locoregional therapy, immunotherapy and the combination in hepatocellular carcinoma: future directions. Liver Cancer. 2019;8:326-40. https://doi.org/10.1159/000494843. 
111. Meng M, Li W, Yang X, Huang G, Wei Z, Ni Y, Han X, Wang J, Ye X Transarterial chemoembolization, ablation, tyrosine kinase inhibitors, and immunotherapy (TATI): a novel treatment for patients with advanced hepatocellular carcinoma. J Cancer Res Ther 2020;16:327-334. doi:10.4103/ jcrt.JCRT_101_20, 2.

112. Li Y, Gao Z-H, Qu X-J. The adverse effects of sorafenib in patients with advanced cancers. Basic Clin Pharmacol Toxicol. 2015;116:216-21. https:// doi.org/10.1111/bcpt.12365.

113. Harnois D. Sorafenib in advanced hepatocellular carcinoma. Yearbook Gastroenterology. 2009;2009:218-9. https://doi.org/10.1016/S0739-593 0(09)79296-7.

114. Cheng A-L, Kang Y-K, Chen Z, Tsao C-J, Qin S, Kim JS, et al. Efficacy and safety of sorafenib in patients in the Asia-Pacific region with advanced hepatocellular carcinoma: a phase III randomised, double-blind, placebocontrolled trial. Lancet Oncol. 2009;10:25-34. https://doi.org/10.1016/S14 70-2045(08)70285-7.

115. Cheng A-L, Finn RS, Qin S, Han K-H, Ikeda K, Piscaglia F, et al. Phase III trial of lenvatinib (LEN) vs sorafenib (SOR) in first-line treatment of patients (pts) with unresectable hepatocellular carcinoma (uHCC). J Clin Oncol. 2017;35: 4001. https://doi.org/10.1200/JCO.2017.35.15_suppl.4001.

116. Meyer T, Fox R, Yt M. Sorafenib in combination with transarterial chemoembolisation in patients with unresectable hepatocellular carcinoma (TACE 2): a randomised placebo-controlled, double-blind, phase 3 trial (vol 2, pg 565, 2017). Lancet Gastroenterol Hepatol. 2017;2:E6.

117. Lencioni R, Llovet JM, Han G, Tak WY, Yang J, Guglielmi A, et al. Sorafenib or placebo plus TACE with doxorubicin-eluting beads for intermediate stage HCC: the SPACE trial. J Hepatol. 2016;64:1090-8. https://doi.org/10.1016/j. jhep.2016.01.012

118. Méndez-Blanco C, Fondevila F, García-Palomo A, González-Gallego J, Mauriz JL. Sorafenib resistance in hepatocarcinoma: role of hypoxia-inducible factors. Exp Mol Med. 2018;50(10):1-9. https://doi.org/10.1038/s12276-018-0159-1.

119. Kudo M, Finn RS, Qin S, Han K-H, Ikeda K, Piscaglia F, et al. Lenvatinib versus sorafenib in first-line treatment of patients with unresectable hepatocellular carcinoma: a randomised phase 3 non-inferiority trial. Lancet. 2018;391: 1163-73. https://doi.org/10.1016/S0140-6736(18)30207-1.

120. Kudo M, Finn RS, Qin S, Han K-H, Ikeda K, Cheng A-L, et al. Analysis of survival and objective response (OR) in patients with hepatocellular carcinoma in a phase III study of lenvatinib (REFLECT). J Clin Oncol. 2019;37: 186. https://doi.org/10.1200/JCO.2019.37.4_suppl.186.

121. Vogel A, Qin S, Kudo M, Hudgens S, Yamashita T, Yoon J-H, et al. Healthrelated quality of life (HRQOL) and disease symptoms in patients with unresectable hepatocellular carcinoma (HCC) treated with lenvatinib (LEN) or sorafenib (SOR). Ann Oncol. 2017;28:v210. https://doi.org/10.1093/a nnonc/mdx369.002.

122. Vogel A, Cervantes A, Chau I, Daniele B, Llovet JM, Meyer T, et al. Hepatocellular carcinoma: ESMO Clinical Practice Guidelines for diagnosis, treatment and follow-up. Ann Oncol. 2018;29:iv238-55. https://doi.org/10.1 093/annonc/mdy308.

123. EASL Clinical Practice Guidelines. Management of hepatocellular carcinoma. J Hepatol. 2018;69:182-236. https://doi.org/10.1016/j.jhep.2018.03.019.

124. John D. Gordan, Erin B. Kennedy, Ghassan K. Abou-Alfa, Muhammad Shaalan beg, Steven T. Brower, Terence P. Gade, et al. Systemic Therapy for Advanced Hepatocellular Carcinoma: ASCO Guideline.

125. US FDA. FDA approves atezolizumab plus bevacizumab for unresectable hepatocellular carcinoma. https://www.fda.gov/drugs/drug-approvals-anddatabases/fda-approves-atezolizumab-plus-bevacizumab-unresectable-hepa tocellular-carcinoma\#: :text=On\%20May\%2029\%2C\%202020\%2C\%2 Othe,not\%20received\%20prior\%20systemic\%20therapy. .

126. Lee MS, Ryoo B-Y, Hsu C-H, Numata K, Stein S, Verret W, et al. Atezolizumab with or without bevacizumab in unresectable hepatocellular carcinoma (GO30140): an open-label, multicentre, phase 1b study. Lancet Oncol. 2020; 21:808-20. https://doi.org/10.1016/S1470-2045(20)30156-X.

127. Qin S, Galle PR, ZhenggangRen Y-HF, Yau T, Wang B, et al. Efficacy and safety of atezolizumab + bevacizumab vs sorafenib in Chinese patients with unresectable HCC in the phase III IMbrave150 study. Liver Cancer Summit. 2020;abstract:OP02-3.

128. Wilhelm SM, Dumas J, Adnane L, Lynch M, Carter CA, Schütz G, et al. Regorafenib (BAY 73-4506): a new oral multikinase inhibitor of angiogenic, stromal and oncogenic receptor tyrosine kinases with potent preclinical antitumor activity. Int J Cancer. 2011;129:245-55. https://doi.org/10.1002/ijc.2 5864 .
129. Bruix J, Qin S, Merle P, Granito A, Huang Y-H, Bodoky G, et al. Regorafenib for patients with hepatocellular carcinoma who progressed on sorafenib treatment (RESORCE): a randomised, double-blind, placebo-controlled, phase 3 trial. Lancet. 2017;389(10064):56-66. https://doi.org/10.1016/S01406736(16)32453-9.

130. Finn RS, Merle P, Granito A, Huang Y-H, Bodoky G, Pracht M, et al. Outcomes of sequential treatment with sorafenib followed by regorafenib for HCC: additional analyses from the phase III RESORCE trial. J Hepatol. 2018;69(2):353-8. https://doi.org/10.1016/j.jhep.2018.04.010.

131. Hoshida Y, Nijman SMB, Kobayashi M, Chan JA, Brunet J-P, Chiang DY, et al. Integrative transcriptome analysis reveals common molecular subclasses of human hepatocellular carcinoma. Cancer Res. 2009;69:7385-92. https://doi. org/10.1158/0008-5472.CAN-09-1089.

132. Abou-Alfa GK, Meyer T, Cheng A-L, El-Khoueiry AB, Rimassa L, Ryoo B-Y, et al. Cabozantinib in patients with advanced and progressing hepatocellular carcinoma. N Engl J Med. 2018;379:54-63. https://doi.org/10.1 056/NEJMoa1717002.

133. Miksad R, Cicin I, Chen Y, Klumpen H, Kim S, Lin Z, et al. Outcomes based on Albumin-Bilirubin (ALBI) grade in the phase 3 CELESTIAL trial of cabozantinib versus placebo in patients with advanced hepatocellular carcinoma (HCC). Ann Oncol. 2019;30:iv134. https://doi.org/10.1093/annonc/ mdz154.021.

134. Syed YY. Ramucirumab: A Review in Hepatocellular Carcinoma. Drugs. 2020; 80:315-22. https://doi.org/10.1007/s40265-020-01263-6.

135. Zhu AX, Park JO, Ryoo B-Y, Yen C-J, Poon R, Pastorelli D, et al. Ramucirumab versus placebo as second-line treatment in patients with advanced hepatocellular carcinoma following first-line therapy with sorafenib (REACH): a randomised, double-blind, multicentre, phase 3 trial. The Lancet Oncology. 2015;16(7):859-70. https://doi.org/10.1016/S1470-2045(15)000509.

136. Zhu AX, Kang Y-K, Yen C-J, Finn RS, Galle PR, Llovet JM, et al. REACH-2: a randomized, double-blind, placebo-controlled phase 3 study of ramucirumab versus placebo as second-line treatment in patients with advanced hepatocellular carcinoma ( $\mathrm{HCC}$ ) and elevated baseline alphafetoprotein (AFP) following first-line sorafenib. JCO. 2018;36(15_suppl):4003. https://doi.org/10.1200/JCO.2018.36.15_suppl.4003.

137. Zhu AX, Kang Y-K, Yen C-J, Finn RS, Galle PR, Llovet JM, et al. Ramucirumab after sorafenib in patients with advanced hepatocellular carcinoma and increased a-fetoprotein concentrations (REACH-2): a randomised, doubleblind, placebo-controlled, phase 3 trial. Lancet Oncol. 2019;20:282-96. https://doi.org/10.1016/S1470-2045(18)30937-9.

138. Yau T, Hsu C, Kim T-Y, Choo S-P, Kang Y-K, Hou M-M, et al. Nivolumab in advanced hepatocellular carcinoma: Sorafenib-experienced Asian cohort analysis. J Hepatol. 2019;71:543-52. https://doi.org/10.1016/j.jhep.2019.05. 014.

139. Finn RS, Ryoo B-Y, Merle P, Kudo M, Bouattour M, Lim HY, et al. Pembrolizumab as second-line therapy in patients with advanced hepatocellular carcinoma in KEYNOTE-240: a randomized, double-blind, phase III trial. J Clin Oncol. 2020;38:193-202. https://doi.org/10.1200/JCO.19. 01307.

140. Kudo M, Lim HY, Cheng A-L, Chao Y, Yau T, Ogasawara S, et al. Phase III study of pembrolizumab (pembro) versus best supportive care (BSC) for second-line therapy in advanced hepatocellular carcinoma (aHCC): KEYNOTE-240 Asian subgroup. JCO. 2020;38(4_suppl):526. https://doi.org/1 0.1200/JCO.2020.38.4_suppl.526.

141. Yau T, Kang Y-K, Kim T-Y, El-Khoueiry AB, Santoro A, Sangro B, et al. Efficacy and safety of Nivolumab plus Ipilimumab in patients with advanced hepatocellular carcinoma previously treated with Sorafenib: the CheckMate 040 randomized Clinical trial. JAMA Oncol. 2020;6:e204564. https://doi.org/1 0.1001/jamaoncol.2020.4564.

142. Tong M, Che N, Zhou L, Luk ST, Kau PW, Chai S, et al. Efficacy of annexin A3 blockade in sensitizing hepatocellular carcinoma to sorafenib and regorafenib. J Hepatol. 2018;69:826-39. https://doi.org/10.1016/j.jhep.2018. 05.034 .

143. Cao L, Cheng H, Jiang Q, Li H, Wu Z. APEX1 is a novel diagnostic and prognostic biomarker for hepatocellular carcinoma. Aging (Albany NY). 2020;12:4573-91. https://doi.org/10.18632/aging.102913.

144. Gu Y, Li X, Bi Y, Zheng Y, Wang J, Li X, et al. CCL14 is a prognostic biomarker and correlates with immune infiltrates in hepatocellular carcinoma. Aging (Albany NY). 2020;12:784-807. https://doi.org/10.18632/a ging.102656. 
145. Huang B, Tian Z-F, Li L-F, Fan Y, Yin H-Y, Li Y, et al. LHX3 is an advancedstage prognostic biomarker and metastatic oncogene in hepatocellular carcinoma. Cancer Biomark. 2019;26(1):31-9. https://doi.org/10.3233/CBM-1 82257.

146. Tang W, Chen Z, Zhang W, Cheng Y, Zhang B, Wu F, et al. The mechanisms of sorafenib resistance in hepatocellular carcinoma: theoretical basis and therapeutic aspects. Signal Transduct Target Ther. 2020;5(1):87. https://doi. org/10.1038/s41392-020-0187-X.

147. Xia S, Pan Y, Liang Y, Xu J, Cai X. The microenvironmental and metabolic aspects of sorafenib resistance in hepatocellular carcinoma. EBioMedicine. 2020;51:102610. https://doi.org/10.1016/j.ebiom.2019.102610.

148. Niu L, Liu L, Yang S, Ren J, Lai PBS, Chen GG. New insights into sorafenib resistance in hepatocellular carcinoma: responsible mechanisms and promising strategies. Biochimica et Biophysica Acta (BBA) - Reviews on Cancer. 2017:1868(2):564-70. https://doi.org/10.1016/j.bbcan.2017.10.002.

149. Rimassa L, Danesi R, Pressiani T, Merle P. Management of adverse events associated with tyrosine kinase inhibitors: improving outcomes for patients with hepatocellular carcinoma. Cancer Treat Rev. 2019;77:20-8. https://doi. org/10.1016/j.ctrv.2019.05.004

\section{Publisher's Note}

Springer Nature remains neutral with regard to jurisdictional claims in published maps and institutional affiliations.

Ready to submit your research? Choose BMC and benefit from:

- fast, convenient online submission

- thorough peer review by experienced researchers in your field

- rapid publication on acceptance

- support for research data, including large and complex data types

- gold Open Access which fosters wider collaboration and increased citations

- maximum visibility for your research: over $100 \mathrm{M}$ website views per year

At $\mathrm{BMC}$, research is always in progress.

Learn more biomedcentral.com/submissions 